\title{
Kinetic equilibrium of iron in the atmospheres of cool dwarf stars
}

\section{The solar strong line spectrum}

\author{
T. Gehren ${ }^{1}$, K. Butler ${ }^{1}$, L. Mashonkina ${ }^{1,2,3}$, J. Reetz ${ }^{1}$, and J. Shi ${ }^{1,4}$ \\ 1 Institut für Astronomie und Astrophysik der Universität München, Universitäts-Sternwarte München, \\ Scheinerstr. 1, 81679 München, Germany \\ 2 Max-Planck Institut für Astrophysik, Karl-Schwarzschild-Str. 1, 85741 Garching, Germany \\ 3 Department of Astronomy, Kazan State University, Kremlevskaya 18, Kazan 8 420008, Russia \\ 4 Beijing Astronomical Observatory, Chinese Academy of Sciences, 100012 Beijing, China
}

Received 16 August 2000 / Accepted 17 November 2000

\begin{abstract}
Line formation calculations of Fe I and FeII in the solar atmosphere are presented for atomic models of iron including all observed terms and line transitions with available $f$-values. Recent improved calculations of Fe I photoionization cross-sections are taken into account, and the influence of collision processes is investigated by comparing synthesized and observed solar line flux profiles. The background is represented by the opacity of all important non-iron elements with iron lines added. Using a representative sample of sufficiently unblended strong Fe I and FeII line profiles, it is evident that line formation is affected by (a) velocity fields and (b) deviations from local thermodynamic equilibrium (NLTE). The calculations are extended to a systematic analysis demonstrating that the ionization equilibrium of iron is recovered for solar parameters $\left(T_{\text {eff }}=5780 \mathrm{~K}, \log g=4.44\right)$ either using the empirical atmospheric model of Holweger \& Müller (1974) and assuming LTE for both Fe I and Fe II or a line-blanketed theoretical atmospheric model with NLTE iron line formation. In the latter case the kinetic equilibrium of Fe I shows a substantial underpopulation of Fe I terms which depends sensitively on both the improved photoionization calculations and the choice of hydrogen collision rates while the Fe II ion is well approximated by LTE. Although the source functions of most of the Fe I lines are nearly thermal, their formation is shifted deeper into the photosphere. NLTE wings of strong Fe I lines are therefore shallower than under the LTE assumption, whereas the cores of the strongest lines display the usual chromospheric contributions. Based on both calculated and laboratory $f$-values the abundances of $37 \mathrm{Fe}$ II lines range between $\log \varepsilon_{\mathrm{Fe} \text { II, } \odot}=7.50$ and 7.56 , depending on atomic and atmospheric models, and those of $117 \mathrm{Fe}$ I lines between $\log \varepsilon_{\mathrm{Fe} \text { II, } \odot}=7.47$ and 7.56 , both with a relatively large scatter of $0.08 \ldots 0.12$. The collisional coupling of Fe I levels is investigated. Electron collisions seem to play only a minor role. Hydrogen collisions are very important between terms of low excitation, and they efficiently thermalize the line source functions but not necessarily the populations of the lower levels that determine the line optical depth. Thermalization of those low-excitation terms that are responsible for most of the lines analyzed is achieved only if the collisional coupling among highly excited Fe I terms and their Fe II parent terms is increased by large factors compared with standard collision rates. Solar flux profiles are reproduced under the assumption of both LTE or NLTE, with nearly all types of atomic and atmospheric models, because the Fe ionization equilibrium depends on the corresponding sets of $f$-values.
\end{abstract}

Key words. line: formation - line: profiles - Sun: photosphere - Sun: abundances

\section{Introduction}

The maximum nuclear binding energy of iron terminates the thermo-nuclear fusion network and thus dominates the pattern of heavy element abundances in most of the stars by an order of magnitude. Although light elements such as $\mathrm{C}, \mathrm{N}, \mathrm{O}, \mathrm{Ne}, \mathrm{Mg}$, and $\mathrm{Si}$ in stars generally are at least as abundant, their influence on the atmospheric structure and on the spectra of cool dwarf stars cannot be compared

Send offprint requests to: T. Gehren, e-mail: gehren@usm.uni-muenchen.de with that of $\mathrm{Fe}$, because their line spectra are less complex by far. The few hundred strong lines of Fe I and in particular Fe II in the near UV are responsible for approximately half of the total line blanketing effect in cool stellar atmospheres (Gehren et al. 1991), and their influence on the atmospheric structure in cool stars is significantly more important than that of the Balmer lines.

The outstanding role of $\mathrm{Fe}$ has also made it a reference element for all astronomical research related to stellar nucleosynthesis and chemical evolution of the Galaxy. Iron has become a synonym for metals in general, and the use 
of $[\mathrm{Fe} / \mathrm{H}]$ as a logarithmic abundance indicator normalized with the corresponding solar abundance often no longer refers to the iron abundance itself but instead some appropriate mix of metal abundances or even an abundance value obtained for a different element. However, iron is not necessarily representative for the light elements since light and heavy elements are synthesized predominantly in stars of substantially different lifetime. Therefore the abundance ratio $[\mathrm{O} / \mathrm{Fe}]$ or $[\mathrm{Mg} / \mathrm{Fe}]$ is often employed to discriminate between stellar populations or stars of different ages. Such an approach requires a very careful examination of both types of line spectra.

The observation of stellar abundances of cool stars is based on spectra of sufficient resolution. Their interpretation, however, relies on our knowledge of stellar atmospheres and line formation. Without doubt there exist a number of problems related to atmospheric line formation that have not yet been solved.

- The requirements for atmospheric models of cool stars are generally specified by very simple assumptions: plane-parallel, hydrostatic stratification in local thermodynamic equilibrium (LTE) with energy transport due to radiation and convection. Calculations including hydrodynamics (Stein \& Nordlund 1998; Nordlund et al. 1997; Spruit et al. 1990; Steffen 1991; Freytag et al. 1996; Asplund et al. 1999) tend to give a better approximation of the atmospheric velocity fields but are not necessarily representative of multi-frequency radiation transport. Ignoring dynamics implies substitution of parameterized non-thermal line-broadening processes generally termed as turbulence (which it is not) on small and large scales;

- The different results of static and dynamic calculations of atmospheric structure are accompanied by the unresolved discrepancy between static theoretical models of the Sun (Gehren et al. 1991) and semi-empirical models such as that of Holweger \& Müller (1974). Though the temperature difference near $\bar{\tau}=1$ between both types of static models is only $150 \mathrm{~K}$, it produces abundance differences of 0.1 to $0.2 \mathrm{dex}$. This discrepancy was not removed with the introduction of improved line opacity data of Kurucz (1992). It may be removed by introducing overshooting of convective motions (Castelli et al. 1997; Kupka 1999; Barklem et al. 2000);

- The solar photospheric Fe abundance itself is under debate (see Kostik et al. 1996, for a discussion). It is obtained assuming LTE from a synthesis of a number of Fe I and Fe II lines of different excitation energy. The discrepancy between the results of the Oxford group $\left(\log \varepsilon_{\mathrm{Fe}, \odot}=7.67\right.$, Blackwell et al. 1995a, 1995b) and the Kiel group $\left(\log \varepsilon_{\mathrm{Fe}, \odot}=7.51\right.$, Holweger et al. $1990,1991,1995)$ is not related to the atmospheric model since both groups use the Holweger-Müller solar model. At least part of the controversy is due to the use of Fe I lines which are much more sensitive to atmospheric parameters than are the Fe II lines. Recent de- terminations are as low as $\log \varepsilon_{\mathrm{Fe} \text { II, } \odot}=7.42$ (Schnabel et al. 1999);

- Throughout the literature abundance analyses of cool stars have been restricted to the assumption of LTE. Only a few exceptions are reported, and it is by no means certain that iron will be found in LTE in all types of stars (Takeda 1991). Most important may be the deviations from local thermodynamic equilibrium in atmospheres of reduced metal abundance such as found in halo turnoff stars or subgiants of the thick disk. The reason for expecting NLTE type equilibria in metal-poor stars is twofold: (a) Interaction between atoms and photons on the one hand and atoms and electrons on the other will be strongly shifted towards radiative processes since, due to the low metal abundance, line blanketing will be less important (this is responsible for a substantial increase in the mean intensity in the near UV). (b) Simultaneously, thermalization due to electron collisions will be less important because the free electron density is correlated with metal abundance in cool stars.

The observed line spectrum in cool and extremely metalpoor stars is almost completely due to FeI, since Fe II lines in the visible are relatively weak. However, Fe I is a minor ionization stage; for a solar-type star near $\bar{\tau}=1$ thermal ionization yields $N(\mathrm{Fe}$ II $) / N(\mathrm{Fe} \mathrm{I}) \approx 10$, and in metal-poor stars as much as $95 \%$ of iron may be ionized. The situation is even more critical in turnoff stars where $N($ Fe II $) / N($ Fe I) easily approaches thermal values of 50 . Consequently, small corrections to the ionization equilibrium can result in very large Fe I abundance corrections for those stars that are most important for our understanding of Galactic evolution.

These considerations have led to a number of analyses of the kinetic equilibrium of iron starting with the work of Lites (1972); Athay \& Lites (1972); Lites (1973); Lites \& Cowley (1974). Steenbock (1985) continued the investigation and came to the conclusion that in particular in the atmospheres of giant stars deviations from LTE must be expected. Even for the Sun he found small NLTE effects showing a clear dependence on the excitation energies of the Fe I levels (Gehren et al. 1991). No deviations from LTE excitation were found for Fe II. Boyarchuk and his co-workers obtained significantly larger deviations from LTE (Boyarchuk et al. 1985; Bikmaev et al. 1990). Their model atoms were, however, less complete than that of Steenbock who used 80 levels for Fe I and 20 levels for Fe II. More recent analyses come from Takeda (1991) who apparently was the first to apply the accelerated lambda iteration (ALI) method to the Fe NLTE line transfer problem. This method allowed a substantial improvement of the model atom considering a total of 280 terms and nearly $5000 b b$ radiative transitions for Fe I and Fe II. Confronted with the problematic determination of Fe I abundances in cool stars two very recent investigations have attacked the NLTE problem with different results. Gratton et al. (1999) used Takeda's "primary" atomic model with slight 
modifications, and they were unaware of the Iron Project photoionization cross-sections of Bautista (1997). Thus their results are quite different from those of Thévenin \& Idiart (1999) who find substantial overionization of Fe I in extremely metal-poor stars. Therefore it is important to explore in much more detail the influence of interaction processes in cool stars of different iron abundance.

In retrospect it is evident that the treatment of the $\mathrm{Fe}$ NLTE problem was always (and still is) dictated by the available computing facilities. The Fe atom is so complex that there is no guarantee that a particular approximation of the term system leads to the desired accuracy. This is emphasized by the recent re-analysis of laboratory Fe I spectra by the Lund group (Nave et al. 1994) which increased the number of observed levels to more than 800 , and the number of lines to 9500. Kurucz's (1992) analysis of the Fe I term system resulted in more than 11400 (mostly predicted) levels with nearly 790000 lines. A systematic evaluation of this data set is still lacking, but even a quick glance at the data reveals that only 25 of the predicted levels of Fe I are below $6.5 \mathrm{eV}$, and the large majority of the lines refers to autoionization transitions. This yields some confidence that the present laboratory results represent the Fe I system with a high degree of completeness at least up to $1 \mathrm{eV}$ below the ionization limit.

Evidence for deviations from LTE also comes from the empirical side. Lyubimkov \& Boyarchuk (1983) found a strong systematic overionization of Fe I in the spectra of F-type supergiants. Magain (1988) and Magain \& Zhao (1996) have claimed evidence for a strong variation of abundances obtained from Fe I lines of different excitation energy in unevolved metal-poor stars. They interpret the results as being due to non-thermal excitation. There is, however, some bias in their results which comes from the requirement that the proper effective temperature of the atmospheric model must be determined. Since their results rely on temperatures obtained from broad-band colours, part of the abundance variation may well be assigned to a systematic offset of effective temperatures.

As outlined above there is more than one reason to investigate the kinetic equilibrium of iron in cool stars. One of the most important considerations bears on the influence of the iron equilibrium on the determination of stellar parameters. Recent analyses based on HipPARCOs parallaxes (Fuhrmann et al. 1997, see Fuhrmann 1998, 2000) give evidence for a significant discrepancy between surface gravities obtained from trigonometric parallaxes and those determined on the basis of the Fe ionization equilibrium assuming LTE. Fuhrmann's results refer to solartype stars in the immediate solar neighbourhood including stars of the thin and thick disk. The differences he finds are systematic, and they seem to support the notion that the kinetic equilibrium of iron may be substantially different from Saha/Boltzmann statistics, particularly in metalpoor stars. Quantitative estimates based on Steenbock's results (Gehren et al. 1991) confirm this idea, but no systematic investigation had been undertaken at that time. Nissen et al. (1997) come to a similar conclusion as far

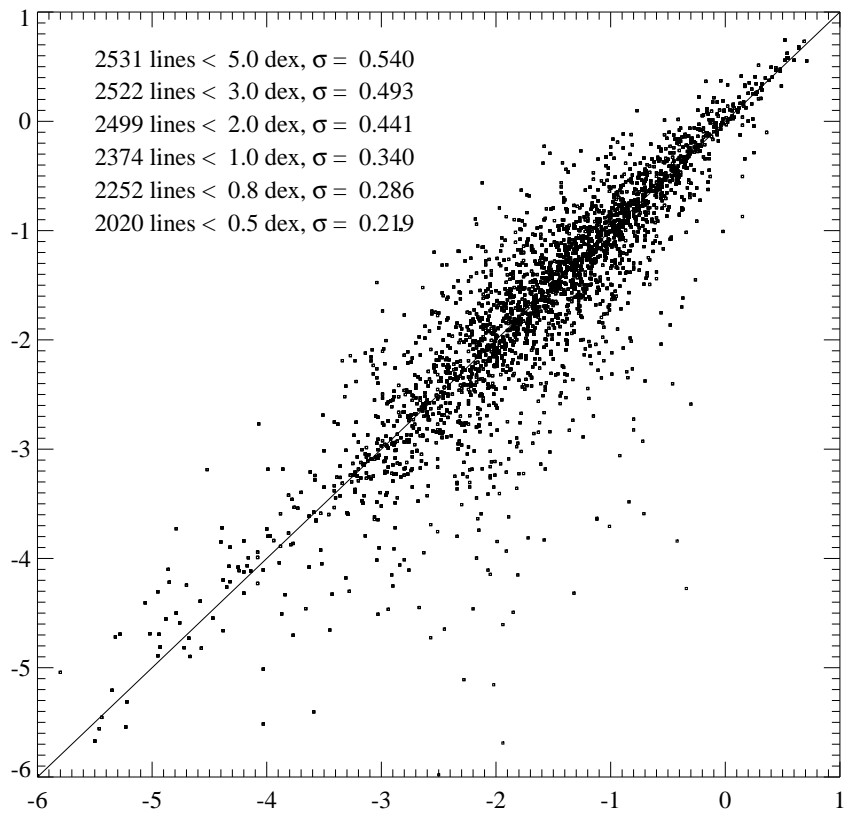

Fig. 1. Comparison of calculated log $g f$-values (Kurucz 1992, $y$ axis) with (experimental) $\log g f$-values ( $x$ axis) given in Table 2 of Nave et al.

as the validity of LTE ionization equilibria in metal-poor dwarfs and subgiants is concerned, and they emphasize the importance of calibrating gravities with the HipPARCOS parallaxes. Allende Prieto et al. (1999) also find systematic differences between spectroscopic and trigonometric gravities, but there seems to be some confusion about the different influences of departures from LTE, effective temperatures and stellar masses. Thus, their trigonometric gravity of Procyon comes out at $\log g=3.73$, assuming a mass of $1.04 M_{\odot}$.

As noted above the surface gravity results depend on the proper effective temperature scale, which has been a matter of controversy between research groups using colours and those preferring (hydrogen) line profiles. The goal of the analysis presented here is therefore to give an account of the solar iron NLTE effects, in particular the Fe II/Fe I ionization equilibrium, and their dependence on atomic interaction processes.

In this first of a series of publications we will constrain our efforts to an analysis of the strong solar iron line spectrum. Due to the availability of accurate parameters, high-resolution spectra and models the Sun is so important that it deserves a separate approach to determine the conditions under which the iron ionization equilibrium is obtained.

The problem is complex since there is no straightforward way to obtain reliable results. Therefore it is convenient to present some kind of standard or reference system both for the atomic and for the atmospheric data. This is presented in the following section where, fortunately, we find that the Fe II ion is well represented by its ground state. The investigation of single interaction 
processes such as photoionization and collisions cannot be done without reference to observed spectra. Thus in Sect. 3 the solar flux spectrum serves as a collection of boundary conditions for photoionization, electron collisions and thermalization, and for the problem of how to deal with hydrogen collisions of which we know little more than that they must exist. The solar spectrum allows us to determine the influence of microturbulence, and that of van der Waals-type damping parameters and compare the latter to the calculations of Anstee \& O'Mara (1991, 1995). It also serves as a base for the analysis of the influence of external broadening processes such as rotation and macroturbulence. Section 4 contains a brief discussion of the results.

\section{Atomic models}

The most extended if not complete data sets describing the iron atom and its first ionization stages are related to Kurucz's (1992) investigation of the iron group elements and to the Iron Project (Hummer et al. 1993). Both aim at describing atomic level systems and interaction crosssections by a theoretical approach. The vast majority of Kurucz's data refers to predicted energy levels the existence of which has not yet been verified by laboratory experiments. The IRON Project started with high ionization stages of $\mathrm{Fe}$ and only recently arrived at Fe I. So there are no $f$-values available as yet. $f$-values for Fe II lines are provided by the Kurucz data base. However, recent calculations of Raassen \& Uylings (1998) present new $f$-values that are systematically smaller than both Kurucz's data and the experimental results of Hannaford et al. (1992) or Schnabel et al. (1999) Damping constants are also a part of a model atom. They have to be used here because metal-poor stars show only Fe I lines in their spectra that are strong in the Sun. As with the IRON Project results, the damping constants of Anstee \& O'Mara (1991, 1995) are based on considerably improved atomic theory.

The atomic models presented in the following subsections have provided the input for the DETAIL NLTE line formation program (Butler \& Giddings 1985), which was used here in a revised version performing radiative transfer with the ALI method.

\subsection{The reference model}

For Fe I the recent experimental analysis of Nave et al. (1994) has approximately doubled empirical knowledge of the Fe I system. 846 levels belonging to 236 terms, and 9759 lines of 2639 multiplets constitute a substantial improvement over the compilation of Sugar \& Corliss (1985) or Fuhr et al. (1988). Theoretical $g f$-values of Kurucz are calculated taking into account configuration interaction which is important in particular for the excited levels of Fe I. The quality of the results is hard to estimate; comparison with experimental data is possible for a subset of 2533 lines of the Nave et al. list, most of which are shown in Fig. 1. The scatter between the two data sets is considerable. Taking the experimental sample as reference the theoretical data show a single line scatter of 0.57 dex which is dominated by quite a small fraction of the lines. Ignoring all lines with deviations above $1 \operatorname{dex}(\approx 10 \%)$ the scatter is reduced to 0.29 dex. The reliability of the calculated $g f$-values is thus roughly characterized by a factor of 2 statistical accuracy only. It is, however, important to note that a minority of the lines compiled by Nave et al. refers to "experimental" data derived from solar spectra assuming a solar iron abundance (see in particular the data of Meylan et al. 1993).

Photoionization is in principle the most important process deciding whether a particular ion tends to be over- or underpopulated in the atmosphere of a cool star. Whenever photoionization is dominant - as is the case for Al (Baumüller et al. 1996, 1997) or Mg (Zhao et al. 1998) - the NLTE effects to be expected are most important in metal-poor stars, and they produce low-excitation line profiles of the neutral atom that are much fainter than under LTE conditions. In the opposite situation collisiondominated ions such as Na (Baumüller et al. 1998) interact with the ion via photon suction that produces downward cascades and over-populates the ground state; $\mathrm{Na}$ lines therefore tend to be stronger in a NLTE environment than under LTE conditions. The results are opposite for abundance determinations with the necessary abundance corrections being positive for the photoionization-type ion and negative for the collision-dominated ion. Exceptions to this rule are found for majority ions such as BaII (Mashonkina et al. 1999) or Eu II (Mashonkina \& Gehren 2000), but the role of collisions and photoionization is very similar in all ions.

The previous approach to photoionization of Fe I was often dictated by missing data being replaced with a hydrogenic approximation of the bf cross-sections (Steenbock 1985; Takeda 1991). For Fe I this is a particularly unsatisfactory situation because the distribution of terms produces a relatively large number of crosssections with ionization edges in the near UV. This is the wavelength region where line blanketing is important, and therefore the photoionization rate, varying with $\int a_{\nu} J_{\nu} \mathrm{d} \nu$, will depend on both the radiation field $J_{\nu}$ and the photoionization cross-section $a_{\nu}$. In order to improve the reliability of the NLTE results recent photoionization calculations of the Iron Project (Bautista 1997) have been used for FeI. Note that the theoretical photoionization cross-sections resulting from close-coupling calculations are larger than the hydrogenic approximations by factors between 10 and 1000. Of course, there is no reason to assume that hydrogenic approximations of low-excitation Fe I states are realistic.

Photoionization is counter-balanced by collisions both by coupling Fe I to the corresponding parent states of Fe II and by cascading recombined electrons to the ground state of Fe I. Collisions can be of any type, but in cool stellar atmospheres electron and neutral hydrogen collisions are the most important. Since they may or may not thermalize the photon flux it is necessary to analyze their influence 

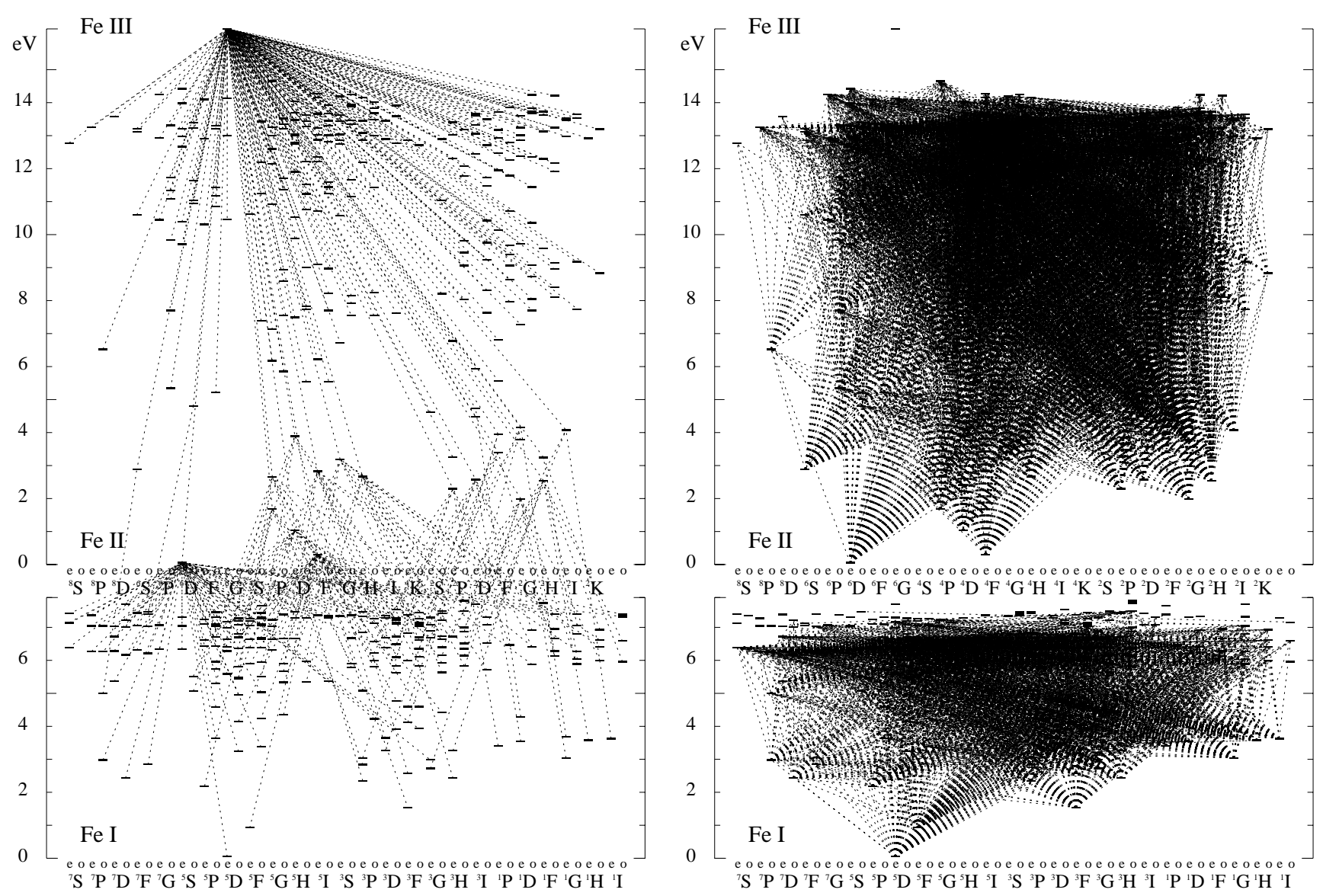

Fig. 2. Grotrian diagram of Fe I and Fe II showing all terms and radiative transitions included in the reference model atom. Left: bf transitions, right: $b b$ transitions

depending on various atmospheric parameters. Assuming LTE, the free electron density is known to depend on temperature and gravity via the Saha equation. Therefore the spectra of hot metal-poor (turnoff) stars display the competing influence of both enhanced UV radiation field and reduced electron collision rates. Similarly, subgiants are expected to show NLTE effects more clearly than do main-sequence stars, since their photospheres are characterized by a reduced electron pressure. However, both predictions depend critically on the ratio of hydrogen to electron collision rates. If neutral particle collision rates become important (mostly due to the large $N_{\mathrm{H}} / N_{\mathrm{e}}$ ratio), potential deviations from LTE are damped by hydrogen collisions. Unfortunately, atomic collisions have not yet been calculated with sufficient accuracy. Thus electron collisions must still be approximated by Coulomb-BornBethe type calculations or the classical Thomson formula (van Regemorter 1962). Therefore, as in all previous calculations the van Regemorter approximation will be used, modified by a Gaunt factor that is $\bar{g}=0.2$ for changing principal quantum number $n$, and 0.7 elsewhere.

Forbidden collisions in our representation follow Allen's (1973) formula for which no experimental verification is available. First explorations of forbidden fine structure transitions have been carried out very recently in the
Iron Project by Pelan \& Berrington (1997) who claim an accuracy for the calculated effective collision strengths (to be compared with the collision strength $\Omega$ in Allen's formula) of between 10 and $20 \%$. Taken at face value this would imply that our assumption of $\Omega=1$ as a general approximation to the forbidden collision strength is not too far from reality. However, there are also hints that source function equality of fine structure transitions sometimes requires considerably stronger collisions.

Ionization by electronic collisions is calculated from Seaton's (1962) classical path approximation with a mean Gaunt factor set equal to $\bar{g}=0.1$ for Fe I and to 0.2 for Fe II. The collision rate is therefore proportional to the photoionization cross-section at the threshold. For all electronic collisions we have investigated the system reaction to a multiplication of all collision rates with a universal enhancement factor (see below).

Finally, the role of collisions with neutral hydrogen atoms has been explored. The basic formula used for allowed transitions is the one originally proposed by Drawin (1968, 1969) as published by Steenbock \& Holweger (1984), however, replacing an obvious misprint in the function $\Psi(W)$.

The Fe II atomic model is completely based on the data calculated by Kurucz (1992). Here, for the sake of 

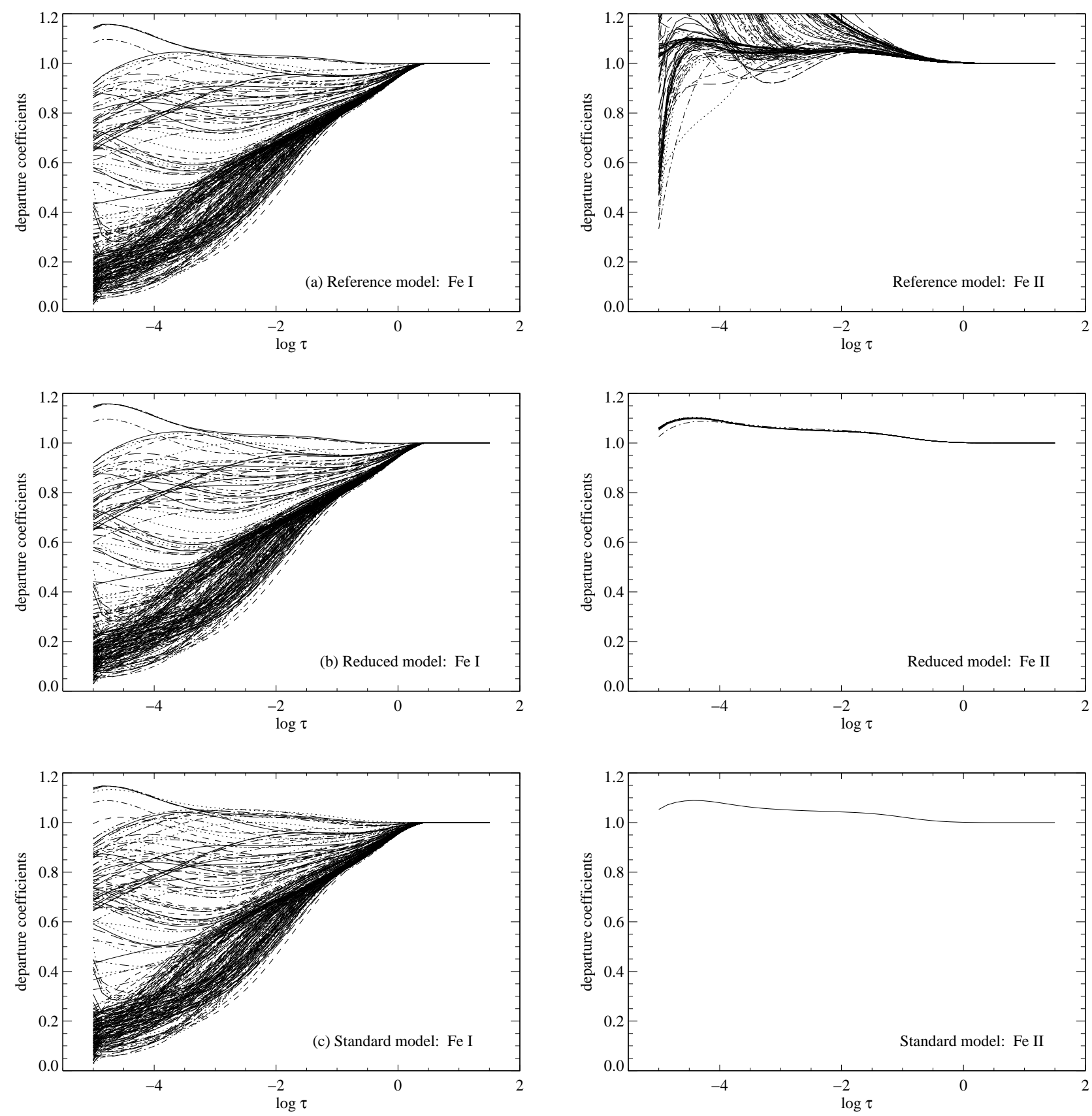

Fig. 3. Departure coefficients $b_{n}$ for all terms of Fe I (left) and Fe II (right) for different atomic models. Photoionization is calculated according to Bautista (1997). No hydrogen collisions are included. Note the invariance of the Fe II metastable terms under different atomic models

simplicity, photoionization to Fe III is calculated in the hydrogenic approximation. This in no way affects our results because Fe III constitutes only an extremely small fraction of the total iron atoms. Collisions of Fe II ions with free electrons are assumed to be twice as efficient compared with Fe I atoms, but the relative excitation of the Fe II levels up to $\sim 6 \mathrm{eV}$ is dominated by line radiation which, as a result of large $f$-values, is optically thick throughout the photosphere. Therefore Fe II tends to follow a Boltzmann equilibrium defined by the local temperature.
The resulting departure coefficients $b_{k}=N_{k} / N_{k}^{\mathrm{LTE}}$ are presented in Fig. 3a, where as in all other figures the Fe II ground state departure coefficient is near its thermal value $\left(b_{\mathrm{Fe} \text { II }} \sim 1\right)$. The neutral iron terms are underpopulated due to photoionization, and the majority of terms with energies below $7 \mathrm{eV}$ are close together while only the high-excitation terms fill the gap in between. In particular we note that the terms above $7 \mathrm{eV}$ do not contribute to the optical solar line spectrum. However, Johansson et al. (1994) and Schoenfeld et al. (1999) have recently 

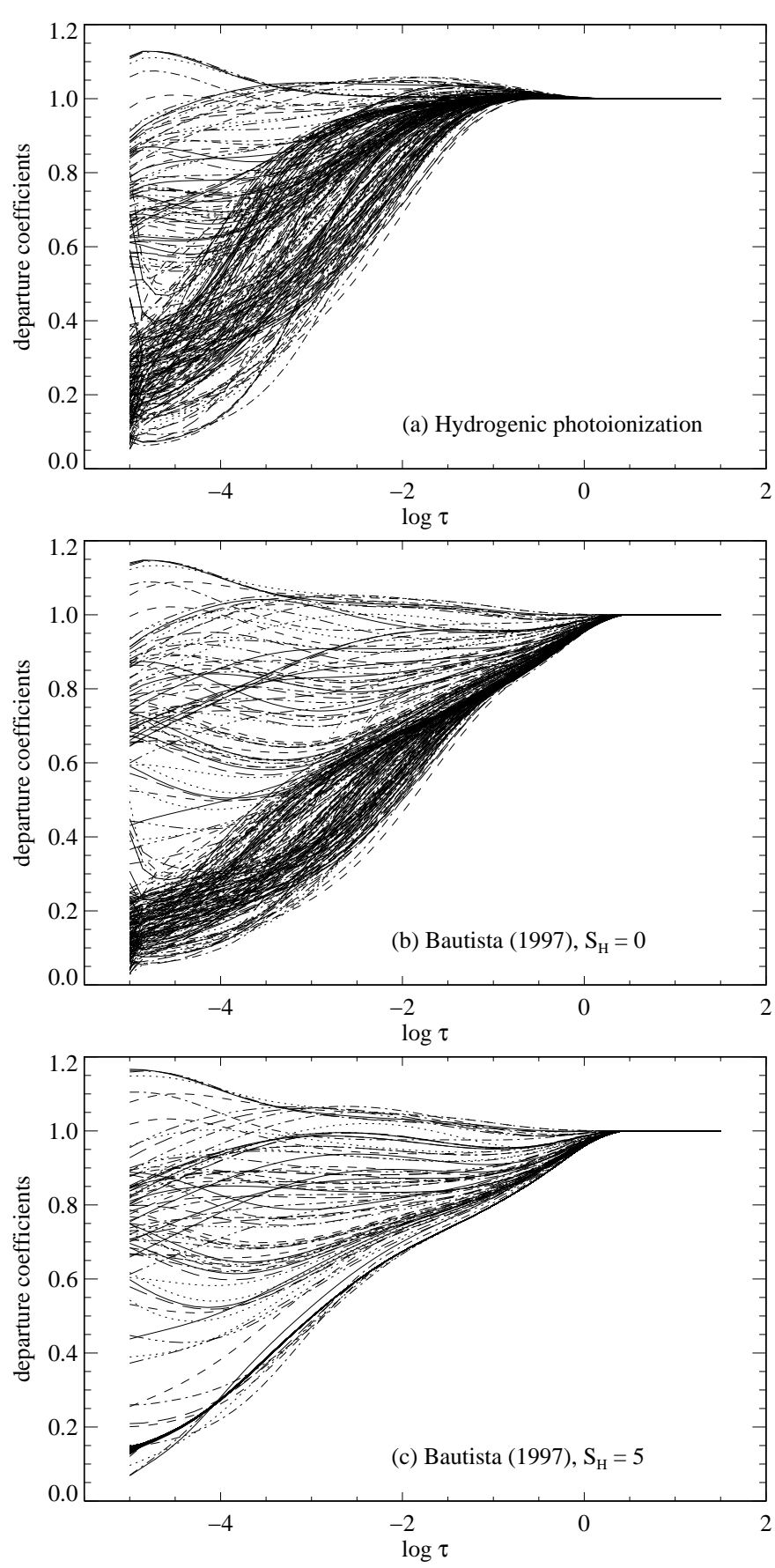

Fig. 4. Departure coefficients $b_{n}$ for all terms of Fe I calculated for the standard atomic model. a) Photoionization calculated with hydrogenic approximation. b) Photoionization cross sections from Bautista (1997). In both cases no hydrogen collisions were included. c) As above, but hydrogen collisions calculated according to the Drawin formula with a multiplication factor 5 . Note the strong relative thermalization of the low-excitation Fe I terms

analyzed the Rydberg multiplets $3 \mathrm{~d}^{6} 4 \mathrm{~s}\left({ }^{6} \mathrm{D}\right) 4 \mathrm{f}-5 \mathrm{~g}, 4 \mathrm{f}-6 \mathrm{~g}$, and $5 \mathrm{~g}-6 \mathrm{~h}$ in the infrared, with terms between 7.0 and $7.65 \mathrm{eV}$. Their results could contribute to the discussion of the upper level thermalization procedure used to force levels near the continuum into LTE (see below).

\subsection{The reduced model}

As was indicated in the preceding subsection the complete reference model consists of a total of 504 terms, 236 of Fe I, 267 of Fe II and 1 of Fe III. In addition to the 503 bf transitions these terms interact through 14908 bb radiative transitions for which $f$-values are known, 4084 of Fe I and 10824 of Fe II. The resulting calculation scheme is very near to prohibitive taking more than $30 \mathrm{~min}$ on a DEC AXP for one ALI cycle; consequently, a completely converged solution requires roughly one day of CPU time. In spite of the considerable overhead we have used this reference atomic model for a number of calculations to examine its convergence properties and make sure that the final solutions are free of numerical problems. However, the restricted range of stellar parameters to be used in this analysis and future investigations - we are basically interested in FGK-type stars - suggests that a reduced atomic model serves our purposes equally well.

It is obvious from Figs. 2 and 3a that the bulk of Fe II terms is neither coupled very closely to the Fe I term system nor is it coupled strongly to the metastable states of Fe II, which are the ones interacting with the Fe I term system through photoionization. Therefore we have defined a considerably reduced atomic model which is devoid of all Fe II terms above $4.7 \mathrm{eV}$. In addition to the complete set of Fe I terms and transitions the reduced model thus involves only those (metastable) terms of the Fe II ion that are parent states to Fe I terms plus the Fe III ground state. Fortunately, this definition removes all $10824 b b$ radiative transitions between Fe II terms. Actually, one single transition $\left(a^{6} \mathrm{D}-\mathrm{z}^{6} \mathrm{D}^{\circ}\right)$ would remain; after a few test calculations we found that this transition could be removed as well since the line is optically thick throughout the atmosphere.

As a result of the above considerations the reduced atomic model consists of 258 terms (236 Fe I, 21 Fe II, $1 \mathrm{Fe}$ III), $236 \mathrm{Fe}$ I $b f$ and $4084 \mathrm{Fe}$ I $b b$ radiative transitions. The reduced model also accounts for the corresponding collisions including the forbidden collisional interaction between the remaining terms of Fe II. It was tested for a number of stellar parameters and found to be representative for the reference model in all cases including the Sun. This is not surprising because the strong deviations from LTE populations found in the Fe II panel of Fig. 3a are all due to highly excited levels that have absolute population densities much smaller than the strongly populated metastable states. In cool stellar atmospheres no visible Fe II lines arise from these levels.

\subsection{The standard model}

Even the reduced atomic model leads to Fe II terms with almost thermalized population densities (see Fig. 3b). Therefore all the parent terms except the Fe II ground state have been removed to leave what we call the standard atomic model. This model thus includes only the 236 Fe I terms plus the Fe II ground state for which we have 
replaced the statistical weight by an appropriate Fe II partition function. Again, this atomic model was tested and the results were compared with those obtained from the reference or the reduced model. In no case did we find substantial changes in the population densities of the Fe I terms (see Fig. 3c). Therefore the final applications have all been calculated with the standard atomic model which may safely be used for all purposes including future analyses of extremely metal-poor stars. All FeII profiles are therefore calculated under LTE assumptions. Since the standard model does not include FeII lines it is necessary to compensate for the missing opacity in the UV; we therefore have sampled LTE Fe line opacities and added them to the background opacity used to calculate photoionization fluxes. This procedure is less extreme than "sampling" opacity distribution functions with a full account of Fe opacities. In particular the strong Fe II lines in the UV are important in blocking the radiation field and reducing the photoionization rates. Our addition of Fe I lines, while not consistent with the model assumptions, proved to be necessary to compensate for the relatively small number of Fe I profile frequencies $(\leq 9$ per transition) dictated by the large number of lines and the finite computer memory. Since the transitions between the Fe I terms used in our standard model do not coincide with the real lines, the blocking effect is perhaps slightly too strong. Thus our model will minimize any NLTE effects.

Although the degree of completeness of Fe I levels up to $7.5 \mathrm{eV}$ may be very high, the results obtained for the population densities or departure coefficients of all the terms above $6.5 \mathrm{eV}$ are not always convincing. Figure $4 \mathrm{~b}$ demonstrates that in the solar atmosphere none of these upper terms couples thermally to the Fe II $a^{6} \mathrm{D}$ ground state, nor is there any strong thermal coupling among the upper terms. For simple reasons of continuity one would expect that thermalization of the upper terms with respect to the continuum ground state becomes more complete the smaller the energy difference. This defect in the atomic model does not depend on the model type (reference, reduced, or standard), and it indicates that either the role of collisions among highly-excited levels must be seriously underestimated or the photoionization crosssections of Bautista (1997) are strongly overestimated (see also Fig. 4a). A first guess therefore would be that hydrogen collisions should play an important role. This is somewhat contradictory because - whatever has been found out for such collisions - the highly-excited transitions should be less affected by hydrogen collisions (Kaulakys 1985; Baumüller \& Gehren 1996, 1997; Baumüller et al. 1998; Zhao et al. 1998). Figure 4c reveals that even the assumption of strong hydrogen collisions does not thermalize the upper levels; instead it leads to a strong relative thermalization among the lower terms.

Since it has long been known (e.g. Athay \& Canfield 1969) that thermalization of fine structure levels may require (forbidden) electron collisions with large enhancement factors we have also investigated here a similarly strong coupling among the upper Fe I terms. The result- ing departure coefficients are shown in Fig. 5 depending on the lower energy limit of the upper terms. For these models the electron collisions are enhanced by very large factors $\left(\sim 10^{10}\right)$ for all transitions between Fe I terms above $6.7,7.0$ or $7.3 \mathrm{eV}$, respectively, including electron collisional ionization to the Fe II ground state. While this is more or less an arbitrary manipulation of the model it has the advantage that it forces the corresponding upper Fe I terms into thermal excitation relative to each other and to the Fe II ground state. Figure 5 nicely shows the tendency towards relative thermal excitation when increasing the range of enhanced electronic collisions to terms of ever lower excitation energy. With the exception of very few terms just above the lower limit of the energy range all terms in the thermalization regime follow the departure coefficients of the FeII ground state. The few terms in Figs. 5b and c with overpopulations relative to Fe II are incompletely coupled to the rest of the terms (similar to the high-excitation terms of Fe II in Fig. 3a). None of the lines arising from these terms is observed in the Sun. Since the relative thermalization of the high-excitation levels starts to converge toward the true ionization limit near $7.3 \mathrm{eV}$, we have selected this value as a lower limit for our model of enhanced electronic collisions. It affects only the uppermost 37 terms of FeI. We note that this choice has the least influence on the departure coefficients of all important Fe I terms with excitation energies below $6.5 \mathrm{eV}$. It therefore will not affect most of the lines detected in cool star spectra as long as the coupling between lower and upper terms of Fe I by hydrogen collisions is small. Whenever hydrogen collisions start to dominate the excitation equilibrium, systematic population differences of 15 to $20 \%$ are encountered (see Sect. 3.2).

The combined influence of thermalization by enhanced electron collisions among the upper terms, and by hydrogen collisions on all terms is now quite different from that of the hydrogen collisions alone, because hydrogen collisions do not couple to the Fe II ground state very efficiently. Using hydrogen collision enhancement factors $S_{\mathrm{H}}$ between 0 and 10 with respect to the formula proposed by Drawin (1968, 1969; see also Steenbock \& Holweger 1984), Fig. 6 shows that in the lower photosphere of the Sun the population densities become increasingly thermalized with respect to Fe II. In fact, $S_{\mathrm{H}}>1$ forces nearly all Fe I terms into LTE in the lower solar photosphere. A reasonable guess of $S_{\mathrm{H}}$ therefore will have to be supported by observed spectra such as that of the Sun or other stars with different abundances and/or surface gravities. We return to the problem of hydrogen collisions below.

\subsection{Comparison with other models}

Atomic models are important whenever NLTE populations or line profiles are calculated. It is therefore necessary to compare our data with those presented in other publications. Thévenin \& Idiart (1999) use a Fe I model with 256 levels (as compared with 236 terms in our Fe I 

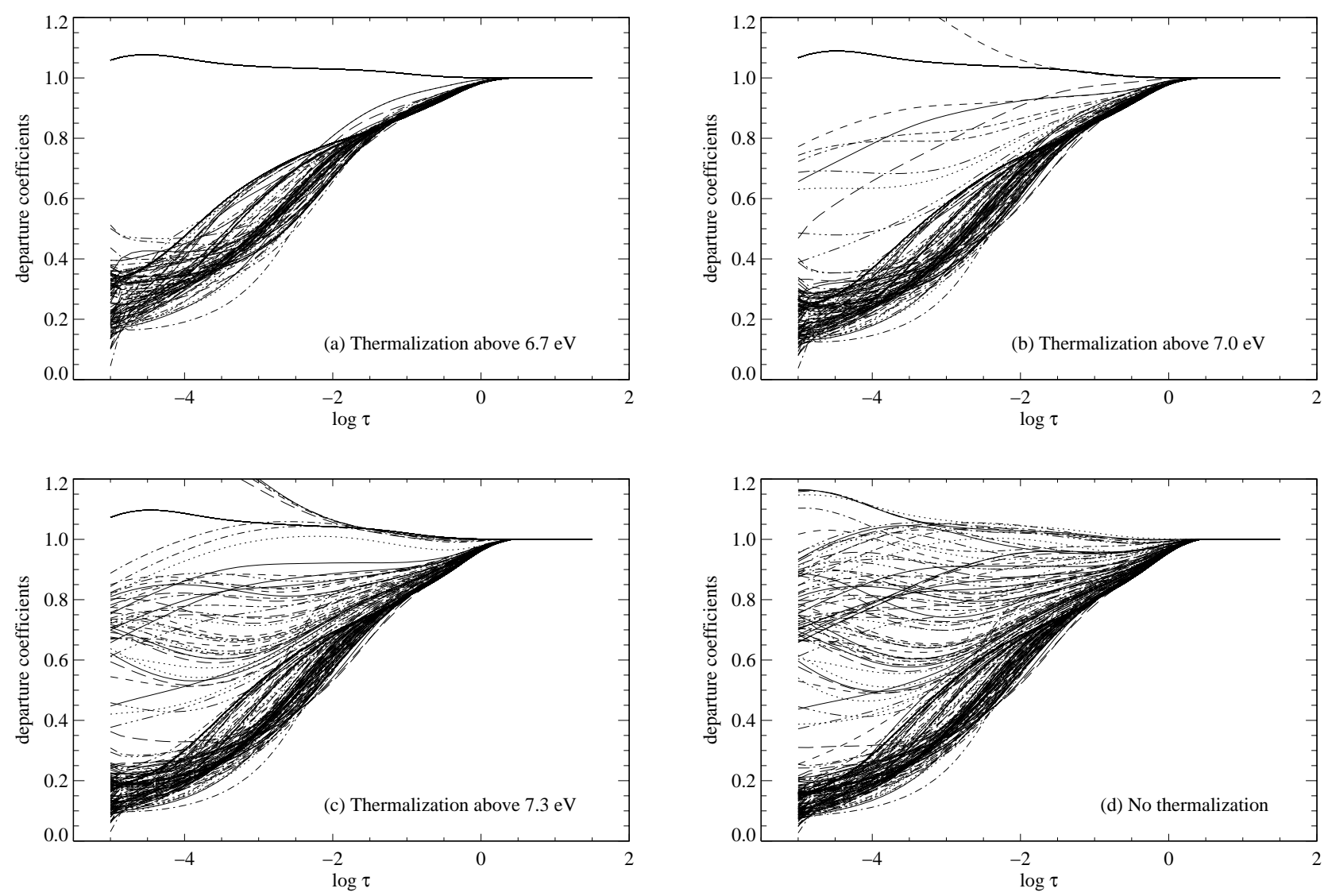

Fig. 5. Departure coefficients $b_{n}$ for all terms of Fe I for different thermalization ranges of the upper levels. Photoionization is calculated according to Bautista (1997). Hydrogen collisions are included with a variable enhancement factor $S_{\mathrm{H}}=2500 \exp \left(n E_{n}\right)$

model). Their fine structure levels result in 2117 Fe I line transitions (as compared with our 4084 Fe I transitions). Although more complete than the atomic model of Gratton et al. (1999), their coverage in excitation energy of the Fe I levels has an upper limit of $6.48 \mathrm{eV}\left(g^{5} \mathrm{D}_{0}\right)$, which is still $1.4 \mathrm{eV}$ below the Fe II ground state. In addition to incompleteness at high energies, a number of configurations are absent, i.e. ${ }^{7} \mathrm{~S},{ }^{7} \mathrm{P},{ }^{7} \mathrm{G},{ }^{5} \mathrm{~S},{ }^{5} \mathrm{H},{ }^{5} \mathrm{I},{ }^{3} \mathrm{I}$, and ${ }^{1} \mathrm{I}$. Whenever collisions start to play a role, the number of channels that transfer electrons between the Fe II ground state and the Fe I levels can be important. The influence of the differences in line transitions is hard to estimate; probably in cool stars the radiative transfer in Fe I $b b$ transitions is less important as long as photoionization dominates. The photoionization was taken from Bautista (1997) as in our investigation. The resulting level populations and line profiles could therefore be very similar to our results except perhaps for a different choice of hydrogen collision rates (see below).

The work of Gratton et al. (1999) refers to quite a different atomic model which was essentially taken from Takeda (1991). Their number of Fe I levels was $50+9$, and the number of FeI line transitions 84; note that these numbers refer to what Takeda called his "primary" set of levels and transitions. Their conclusion that Fe I should be well represented by 59 levels may be somewhat pre- mature. In particular the cascade of recombining electrons requires efficient channels with excitation energies well above $7 \mathrm{eV}$ (Gehren et al. 1991). To establish such a connection, Gratton et al. used 9 additional highlyexcited levels that carried a total statistical weight of only $\sim 1000$. This is significantly less than should be assigned to such hypothetical levels, but in view of their factor of 3 incompleteness of levels below $6.9 \mathrm{eV}$ the upper levels may not be very important. The most important difference with respect to Thévenin \& Idiart's and our present atomic model is, however, the treatment of photoionization. Gratton et al. were obviously unaware of Bautista's work, and they used cross-sections from different sources, all of them one or two orders of magnitude smaller than those of Bautista. Simultaneously, their hydrogen collision rates were increased from Drawin's $(1968,1969)$ formula by a factor of $\sim 30$. Thus it is not surprising that they find nearly thermalized populations throughout the photosphere.

\section{The solar Fe spectrum}

Our atomic model of Fe is subject to large sources of uncertainty. While the cross-sections for radiative interaction seem to be quite reliable, those for collisions are virtually unknown. As usual this ignorance is hidden behind some 

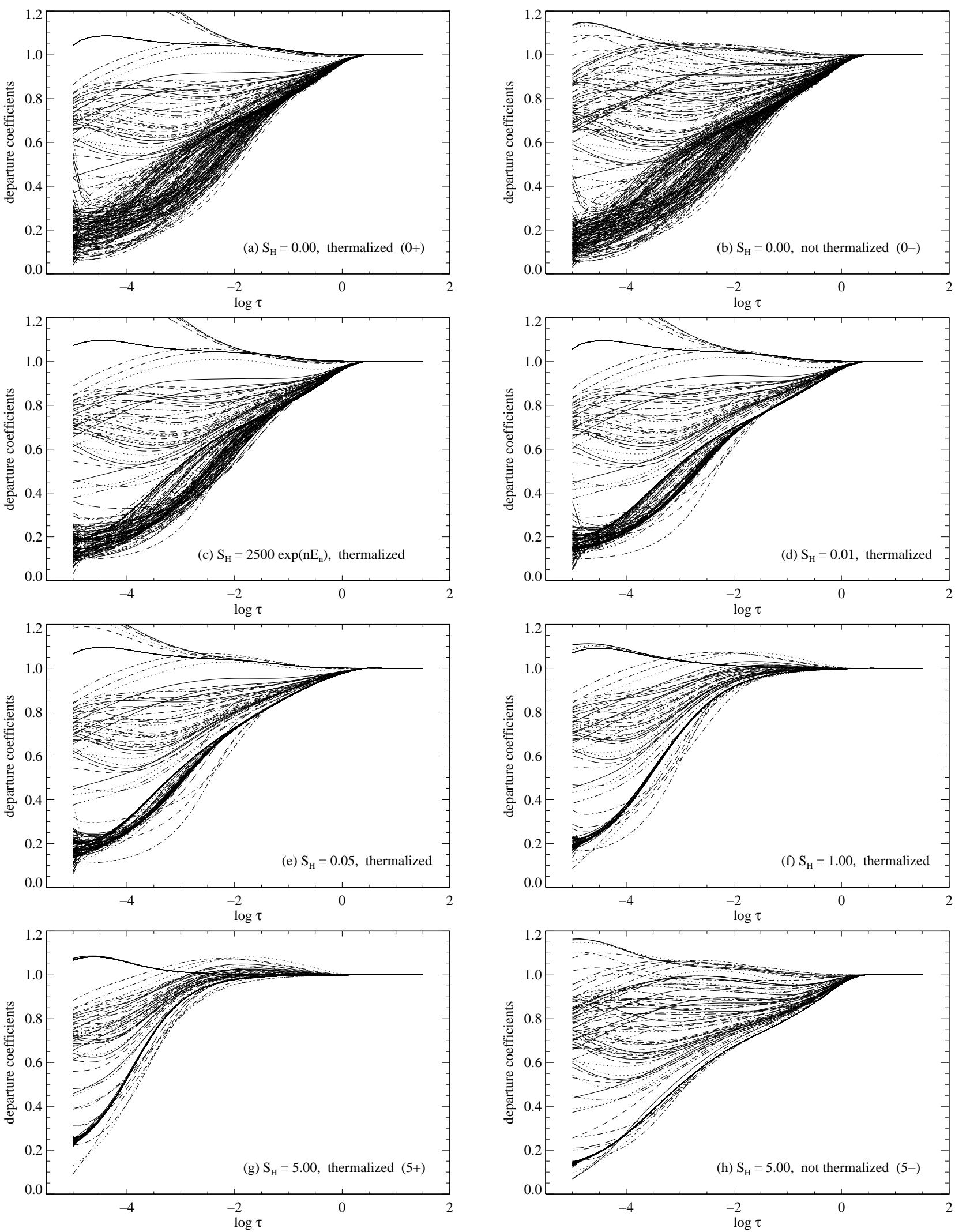

Fig. 6. Departure coefficients $b_{n}$ for all terms of Fe I for different hydrogen collision enhancement factors. Photoionization is calculated according to Bautista (1997); thermalization is enforced for all Fe I terms above 7.3 eV except where noted otherwise. Note that the line source function is thermalized already for relatively small collision enhancement factors, and that thermalization of upper level populations ties to the low-energy levels at moderate optical depths 
scaling rules that allow collision rate coefficients to be calculated as a function of temperature as required for our statistical equilibrium system. The principal criticism to this approach is that one cannot estimate the reliability of the resulting population densities, and the usual response to that criticism is that it is hoped that the statistical approach is insensitive to the fine detail of collisional interactions as much as possible. The very large number of free parameters makes it impossible to evaluate the influence of each interaction rate separately.

For comparison with observed data a total of 154 lines has been chosen to represent the much more complex model. 37 of these lines are due to Fe II and thus synthesized under LTE conditions, the 117 Fe I lines are all synthesized from model NLTE populations. Selection criteria were as follows: a) sufficient representation of excitation energies, b) mostly strong lines to guarantee detectability even in extremely metal-poor stars, and c) minimum blend problems. Not all criteria could always be fulfilled simultaneously. In particular, the first two criteria are hardly fulfilled for Fe II. A list of all lines is given in Tables 1 and 2 . As far as possible the data (including $f$-values) have been taken from Nave et al. (1994) for Fe I, and from Raassen \& Uylings (1998) and Kurucz (1992) for Fe II. Given an atmospheric model this leaves Fe abundances, damping parameters, and to some extent the non-thermal broadening velocities (micro- and macroturbulence) as adjustable parameters. We comment on these parameters below.

- In our analysis we investigate line formation in both empirical and theoretical model atmospheres. The empirical model is that of Holweger \& Müller (HM, 1974) which is known to give an excellent fit to the centreto-limb variation of the continuum radiation. Since the HM model is based on many neutral Fe lines assuming LTE, it may not lead to an equally good fit for NLTE line formation in some of the lines. Therefore our line formation calculations for the HM model atmosphere are restricted to the LTE assumption. The theoretical model $(\mathrm{TH})$ is the typical line-blanketed solar model making use of Kurucz's (1992) opacity distribution functions (ODF), however, rescaling the Fe abundance to the solar system meteoritic value, $\log \varepsilon_{\mathrm{Fe}}=7.51$. The $\mathrm{TH}$ model is based on the radiative transfer code using variable Eddington factors of Mihalas et al. (1975), with temperature correction according to radiative-convective equilibrium. Radiative transfer is modulated by $b f$ and $f f$ absorption from all important ionization stages. In particular, we have included the recent Fe I bf absorption cross-sections of Bautista (1997). Optionally, the $b b$ absorption (line blanketing) processes are included either by means of ODFs or by opacity sampling based on essentially the same line lists. 1D convection is modelled either with the mixing-length approach of Böhm-Vitense (1958) or with a slightly modified version of Canuto \& Mazzitelli (1992), both codes requiring their own mixing-length calibration $\left(\ell / H_{\mathrm{p}}=0.5\right.$ or 0.8 , respectively).
The solar model atmosphere used here (ODFs and Böhm-Vitense convection) is within $30 \mathrm{~K}$ the same as both the rescaled Kurucz model and our variant with opacity sampling assuming an adequate choice of the mixing-length parameter; this agreement justifies the abundance rescaling procedure. We note that the Bautista cross-sections somewhat relax the missing UV opacity problem but the TH model is still unable to reproduce the observed center-to-limb variation of the solar intensity spectrum.

The main difference between the empirical and the theoretical model is the temperature stratification, the HM model being $\sim 100 \ldots 150 \mathrm{~K}$ hotter than the theoretical model at optical depths $\log \bar{\tau}<-0.5$;

- The values of the $\mathrm{Fe}$ abundances reproduced in Tables 1 and 2 are to be understood in the first instance as a measure of uncertainty of the $f$-values which, for the sake of simplicity, have all been adopted in a rather uncritical sense. There are also unresolved blends which have been fitted interactively taking into account their influence on both line core and wings of individual lines. The fits depend on the type of atmospheric model used; results are given for both models;

- Collisional damping depends on stellar atmospheric temperatures. In cool solar-type stars van der Waals damping is the most important atomic broadening process for neutral iron lines while in hot stars the linear Stark effect and radiation damping also contribute. For all atoms other than hydrogen resonance broadening can be neglected because of the low number densities of the atoms. The quadratic Stark effect is important for transitions between levels in simple atoms (such as $\mathrm{NaI}$ or $\mathrm{MgI}$ ). In more complex ions such as Fe I it is generally negligible, or the very small amounts of damping due to the electric microfield can be represented by the temperature dependence of the van der Waals damping process which is therefore the only damping contribution to the wings of strong Fe I lines that is considered here.

Van der Waals damping parameters have been calculated for different degrees of complexity. Based on elementary quantum mechanics Unsöld (1968, Eqs. (54, 82) has described collisions between hydrogen and metal atoms by a van der Waals-type interaction potential that produces an $r^{-6}$ dependence of the line frequency with an interaction constant $C_{6}$ proportional to the polarizability $\alpha$ of the hydrogen atom and to the mean square radius $\overline{r_{\mathrm{e}}^{2}}$ of the quantum state involved. This relatively simple approach has been used with great success by many astrophysicists. The resulting damping constants are useful even for complex atoms, and they fit to the observed profiles of many lines found in the solar spectrum. Kurucz (1992) has published slightly improved interaction constants based on a better description of the atomic configuration. However, for most of the Fe I lines the differences with respect to the Unsöld formula are small. Recent improvements come from Anstee \& O'Mara (1991, 1995) who 
Table 1. 37 Fe II lines selected as solar abundance reference. Sources of $f$-values are Raassen \& Uylings (1, 1998), Kurucz (2, 1992), Hannaford et al. (3, 1992), and Schnabel et al. (4, 1999). Abundances in the columns on the right refer to LTE line syntheses based on the empirical solar model of Holweger \& Müller (1974, HM), and on a line-blanketed solar model (TH), assuming a microturbulence velocity of $\xi_{\mathrm{t}}=1.0$ and $0.85 \mathrm{~km} \mathrm{~s}^{-1}$, respectively. They are obtained from optimized profile fits allowing for some individual variation of van der Waals damping constants $\Delta \log C_{6}$ and the iron abundance, where $\log C_{6}$ refers to Kurucz's (1992) line list

\begin{tabular}{|c|c|c|c|c|c|c|c|c|c|c|c|}
\hline \multirow[t]{2}{*}{ Mult } & \multirow{2}{*}{\multicolumn{2}{|c|}{ Transition }} & \multirow[t]{2}{*}{$\lambda[\AA]$} & \multirow[t]{2}{*}{$E[\mathrm{eV}]$} & \multirow[t]{2}{*}{$\log g f$} & \multirow[t]{2}{*}{$\log C_{6}$} & \multicolumn{2}{|c|}{$\Delta \log C_{6}$} & \multicolumn{2}{|c|}{$\log \varepsilon_{\mathrm{Fe} \text { II }}$} & \multirow[t]{2}{*}{ Source } \\
\hline & & & & & & & HM & $\mathrm{TH}$ & HM & TH & \\
\hline 27 & $\mathrm{~b}^{4} \mathrm{P}_{0.5}$ & $-\mathrm{z}^{4} \mathrm{D}_{1.5}^{\mathrm{o}}$ & 4416.825 & 2.77 & -2.53 & -32.18 & & +0.40 & 7.39 & 7.39 & 2 \\
\hline 27 & $b^{4} \mathrm{P}_{2.5}$ & $-\mathrm{z}^{4} \mathrm{D}_{3.5}^{\mathrm{o}}$ & 4233.170 & 2.57 & -1.91 & -32.18 & & +0.40 & 7.47 & 7.44 & 3 \\
\hline 35 & $\mathrm{~b}^{4} \mathrm{~F}_{2.5}$ & $-\mathrm{z}^{6} \mathrm{~F}_{1.5}^{\mathrm{o}}$ & 5136.802 & 2.83 & -4.37 & -32.18 & & +0.40 & 7.49 & 7.45 & 1 \\
\hline 35 & $\mathrm{~b}^{4} \mathrm{~F}_{4.5}$ & $-\mathrm{z}^{6} \mathrm{~F}_{4.5}^{\mathrm{o}}$ & 5132.669 & 2.79 & -4.13 & -32.18 & & +0.40 & 7.52 & 7.48 & 1 \\
\hline 35 & $\mathrm{~b}^{4} \mathrm{~F}_{4.5}$ & $-\mathrm{z}^{6} \mathrm{~F}_{3.5}^{\mathrm{o}}$ & 5100.664 & 2.79 & -4.22 & -32.18 & & +0.40 & 7.57 & 7.53 & 1 \\
\hline 36 & $\mathrm{~b}^{4} \mathrm{~F}_{4.5}$ & $-\mathrm{z}^{6} \mathrm{P}_{3.5}^{\mathrm{o}}$ & 4993.350 & 2.79 & -3.70 & -32.18 & & & 7.49 & 7.46 & 1 \\
\hline 37 & $\mathrm{~b}^{4} \mathrm{~F}_{4.5}$ & $-\mathrm{z}^{4} \mathrm{~F}_{4.5}^{\mathrm{o}}$ & 4629.340 & 2.79 & -2.31 & -32.18 & & & 7.45 & 7.45 & 2 \\
\hline 37 & $\mathrm{~b}^{4} \mathrm{~F}_{2.5}$ & $-\mathrm{z}^{4} \mathrm{~F}_{3.5}^{\mathrm{o}}$ & 4582.835 & 2.83 & -3.22 & -32.18 & & & 7.51 & 7.51 & 1 \\
\hline 37 & $\mathrm{~b}^{4} \mathrm{~F}_{3.5}$ & $-\mathrm{z}^{4} \mathrm{~F}_{3.5}^{\mathrm{o}}$ & 4555.892 & 2.82 & -2.33 & -32.18 & & & 7.47 & 7.47 & 2 \\
\hline 37 & $\mathrm{~b}^{4} \mathrm{~F}_{2.5}$ & $-\mathrm{z}^{4} \mathrm{~F}_{2.5}^{\mathrm{o}}$ & 4515.338 & 2.83 & -2.47 & -32.18 & +0.30 & +0.30 & 7.43 & 7.43 & 2 \\
\hline 37 & $\mathrm{~b}^{4} \mathrm{~F}_{1.5}$ & $-\mathrm{z}^{4} \mathrm{~F}_{1.5}^{\mathrm{o}}$ & 4491.404 & 2.84 & -2.76 & -32.18 & +0.40 & +0.40 & 7.58 & 7.53 & 1 \\
\hline 38 & $\mathrm{~b}^{4} \mathrm{~F}_{3.5}$ & $-\mathrm{z}^{4} \mathrm{D}_{3.5}^{\mathrm{o}}$ & 4620.527 & 2.82 & -3.29 & -32.18 & +0.40 & +0.40 & 7.45 & 7.45 & 1 \\
\hline 38 & $\mathrm{~b}^{4} \mathrm{~F}_{2.5}$ & $-\mathrm{z}^{4} \mathrm{D}_{2.5}^{\mathrm{o}}$ & 4576.336 & 2.83 & -2.96 & -32.18 & +0.40 & +0.40 & 7.49 & 7.49 & 1 \\
\hline 38 & $\mathrm{~b}^{4} \mathrm{~F}_{1.5}$ & $-\mathrm{z}^{4} \mathrm{D}_{0.5}^{\mathrm{o}}$ & 4508.285 & 2.84 & -2.34 & -32.18 & +0.40 & +0.40 & 7.48 & 7.46 & 1 \\
\hline 40 & $\mathrm{a}^{6} \mathrm{~S}_{2.5}$ & $-\mathrm{z}^{6} \mathrm{D}_{3.5}^{\mathrm{o}}$ & 6516.086 & 2.88 & -3.46 & -32.11 & & & 7.66 & 7.69 & 1 \\
\hline 40 & $\mathrm{a}^{6} \mathrm{~S}_{2.5}$ & $-\mathrm{z}^{6} \mathrm{D}_{2.5}^{\mathrm{o}}$ & 6432.680 & 2.88 & -3.71 & -32.11 & & & 7.60 & 7.63 & 1 \\
\hline 40 & $\mathrm{a}^{6} \mathrm{~S}_{2.5}$ & $-\mathrm{z}^{6} \mathrm{D}_{1.5}^{\mathrm{o}}$ & 6369.463 & 2.88 & -4.25 & -32.11 & & & 7.59 & 7.59 & 1 \\
\hline 41 & $\mathrm{a}^{6} \mathrm{~S}_{2.5}$ & $-\mathrm{z}^{6} \mathrm{~F}_{3.5}^{\mathrm{o}}$ & 5284.109 & 2.88 & -3.30 & -32.11 & & & 7.70 & 7.70 & 2 \\
\hline 42 & $\mathrm{a}^{6} \mathrm{~S}_{2.5}$ & $-\mathrm{z}^{6} \mathrm{P}_{3.5}^{\mathrm{o}}$ & 5169.047 & 2.88 & -1.30 & -32.11 & +0.30 & +0.10 & 7.54 & 7.50 & 2 \\
\hline 42 & $\mathrm{a}^{6} \mathrm{~S}_{2.5}$ & $-\mathrm{z}^{6} \mathrm{P}_{2.5}^{\mathrm{o}}$ & 5018.447 & 2.88 & -1.40 & -32.11 & +0.20 & & 7.65 & 7.62 & 2 \\
\hline 42 & $a^{6} S_{2.5}$ & $-\mathrm{z}^{6} \mathrm{P}_{1.5}^{\mathrm{o}}$ & 4923.931 & 2.88 & -1.56 & -32.11 & +0.30 & +0.20 & 7.61 & 7.53 & 2 \\
\hline 43 & $\mathrm{a}^{6} \mathrm{~S}_{2.5}$ & $-\mathrm{z}^{4} \mathrm{D}_{2.5}^{\mathrm{o}}$ & 4656.981 & 2.88 & -3.66 & -32.11 & & & 7.56 & 7.56 & 1 \\
\hline 46 & $a^{4} G_{4.5}$ & $-\mathrm{z}^{6} \mathrm{~F}_{3.5}^{\mathrm{o}}$ & 6084.110 & 3.19 & -3.89 & -32.19 & & & 7.58 & 7.58 & 1 \\
\hline 46 & $\mathrm{a}^{4} \mathrm{G}_{5.5}$ & $-\mathrm{z}^{6} \mathrm{~F}_{4.5}^{\mathrm{o}}$ & 5991.378 & 3.14 & -3.66 & -32.19 & & & 7.59 & 7.59 & 1 \\
\hline 48 & $\mathrm{a}^{4} \mathrm{G}_{3.5}$ & $-\mathrm{z}^{4} \mathrm{D}_{3.5}^{\mathrm{o}}$ & 5414.075 & 3.21 & -3.65 & -32.19 & & & 7.58 & 7.58 & 1 \\
\hline 48 & $\mathrm{a}^{4} \mathrm{G}_{4.5}$ & $-\mathrm{z}^{4} \mathrm{D}_{3.5}^{\mathrm{o}}$ & 5362.867 & 3.19 & -2.74 & -32.19 & & & 7.69 & 7.69 & 2 \\
\hline 48 & $a^{4} G_{2.5}$ & $-\mathrm{z}^{4} \mathrm{D}_{1.5}^{\mathrm{o}}$ & 5264.808 & 3.22 & -3.23 & -32.19 & & & 7.64 & 7.64 & 4 \\
\hline 49 & $\mathrm{a}^{4} \mathrm{G}_{4.5}$ & $-\mathrm{z}^{4} \mathrm{~F}_{4.5}^{\mathrm{o}}$ & 5425.257 & 3.19 & -3.38 & -32.19 & & & 7.62 & 7.62 & 1 \\
\hline 49 & $\mathrm{a}^{4} \mathrm{G}_{3.5}$ & $-\mathrm{z}^{4} \mathrm{~F}_{3.5}^{\mathrm{o}}$ & 5325.560 & 3.21 & -3.32 & -32.19 & & & 7.62 & 7.61 & 1 \\
\hline 49 & $\mathrm{a}^{4} \mathrm{G}_{5.5}$ & $-\mathrm{z}^{4} \mathrm{~F}_{4.5}^{\mathrm{o}}$ & 5316.618 & 3.14 & -1.93 & -32.19 & +0.30 & +0.30 & 7.54 & 7.56 & 2 \\
\hline 49 & $\mathrm{a}^{4} \mathrm{G}_{3.5}$ & $-\mathrm{z}^{4} \mathrm{~F}_{2.5}^{\mathrm{o}}$ & 5234.631 & 3.21 & -2.27 & -32.19 & +0.30 & +0.30 & 7.54 & 7.56 & 1 \\
\hline 49 & $\mathrm{a}^{4} \mathrm{G}_{2.5}$ & $-\mathrm{z}^{4} \mathrm{~F}_{1.5}^{\mathrm{o}}$ & 5197.575 & 3.22 & -2.34 & -32.19 & +0.30 & +0.30 & 7.55 & 7.55 & 1 \\
\hline 74 & $\mathrm{~b}^{4} \mathrm{D}_{3.5}$ & $-\mathrm{z}^{4} \mathrm{P}_{2.5}^{\mathrm{o}}$ & 6456.383 & 3.89 & -2.18 & -32.18 & & & 7.56 & 7.61 & 1 \\
\hline 74 & $\mathrm{~b}^{4} \mathrm{D}_{2.5}$ & $-\mathrm{z}^{4} \mathrm{P}_{2.5}^{\mathrm{o}}$ & 6416.928 & 3.87 & -2.87 & -32.18 & & & 7.70 & 7.73 & 1 \\
\hline 74 & $\mathrm{~b}^{4} \mathrm{D}_{2.5}$ & $-\mathrm{z}^{4} \mathrm{P}_{1.5}^{\mathrm{o}}$ & 6247.560 & 3.87 & -2.43 & -32.18 & & & 7.57 & 7.61 & 1 \\
\hline 74 & $\mathrm{~b}^{4} \mathrm{D}_{0.5}$ & $-\mathrm{z}^{4} \mathrm{P}_{1.5}^{\mathrm{o}}$ & 6239.944 & 3.87 & -3.57 & -32.18 & & & 7.60 & 7.59 & 1 \\
\hline 74 & $b^{4} D_{0.5}$ & $-\mathrm{z}^{4} \mathrm{P}_{0.5}^{\mathrm{o}}$ & 6149.250 & 3.87 & -2.84 & -32.18 & & & 7.58 & 7.61 & 1 \\
\hline \multirow{2}{*}{\multicolumn{3}{|c|}{ all lines }} & & & & & & & 7.56 & 7.55 & \\
\hline & & & & & & & & & \pm 0.08 & \pm 0.08 & \\
\hline
\end{tabular}




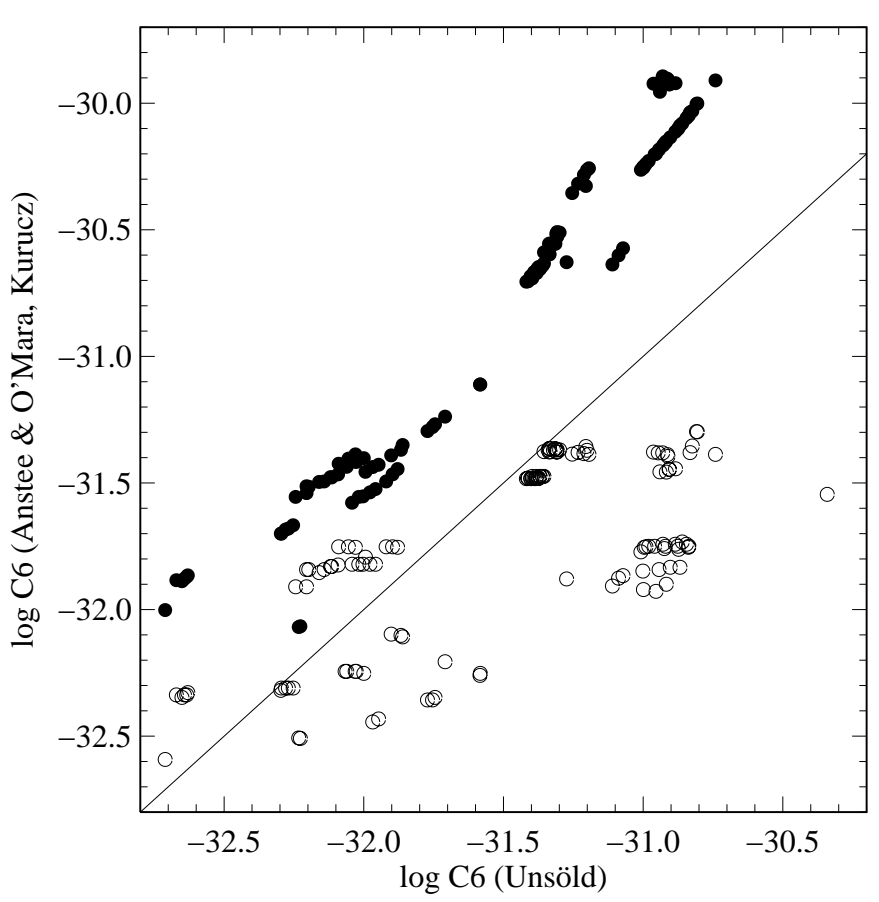

Fig. 7. Van der Waals damping parameter $\log C_{6}$ for Fe I lines as calculated by Anstee \& O'Mara (filled circles, 1991, 1995) and Kurucz (open circles, 1992), compared with the result of Unsöld's (1968) approximation. Note that Anstee \& O'Mara's results are systematically higher by $\approx 0.5 \ldots 0.7 \mathrm{dex}$

calculate explicitly the interaction energies for system orientations thus producing tables of line-broadening cross-sections for different types of transitions (s-p, p-s, p-d, d-p etc.) and of velocity exponents specifying the temperature dependence of the collision processes. The difference between the standard formula of Unsöld and the calculations of Kurucz and Anstee \& O'Mara are evident from Fig. 7 . For most of the lines the latter $C_{6}$ values are $\approx 0.6$ to 0.7 dex higher, and they fit solar line profiles for the theoretical atmospheric models without much modification. The actual precision of such calculations is, however, still under debate (Leininger et al. 2000), and selected multiplets may require corrections. For simplicity we have used in Table 2 Anstee \& O'Mara's results for Fe I without modification in both empirical and theoretical atmospheric models. Table 1 instead indicates the corrections with respect to the Kurucz data that were applied to improve the final profile fits of Fe II lines. As was mentioned above, the multiplets were primarily selected to show lines strong enough to be detected even in extremely metal-poor stars. Thus it was not always possible to determine the relative abundance scale from faint lines in a multiplet. As a consequence the ambiguity between fitting $\log C_{6}$ and $\log g f \varepsilon$ could not always be resolved;

- The shortcomings of modelling the solar atmosphere as a plane-parallel horizontally homogeneous stratification are most obvious when velocity fields are introduced. If line profiles are analyzed carefully, it becomes immediately evident that different lines seem to

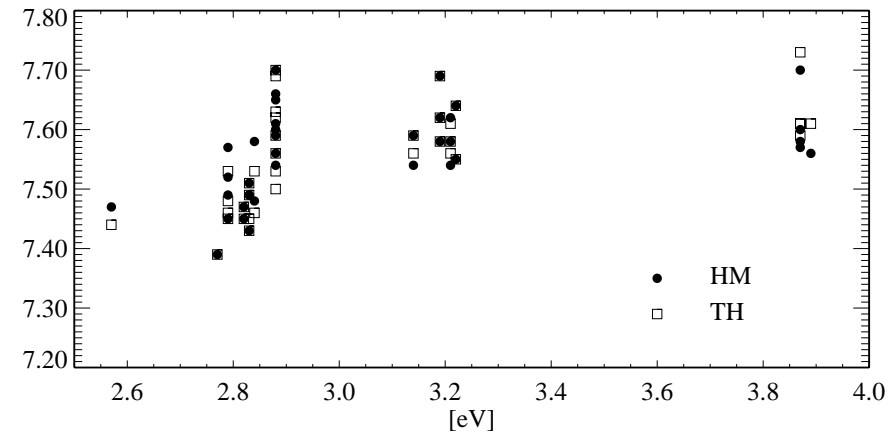

Fig. 8. Fe II abundances obtained for empirical and theoretical atmospheric models plotted against the lower level excitation energy

require different micro- and/or macroturbulent broadening velocities. If solar intensities are compared with theoretical profiles, lines observed at the limb require higher velocities than at the Sun centre. Observed flux profiles require different angular mean microturbulent velocities mostly depending on mean depth of line core formation. Since Holweger (1967) it has been known that a depth-dependent microturbulence distribution arises that essentially hides the real 3D hydrodynamics. A corresponding $\xi_{\mathrm{t}}(\bar{\tau})$ is part of the HM model, and a similar distribution would be adequate for the TH model. In other stars it is, however, often not possible to determine more than a single depth-independent parameter for all Fe lines. Therefore in the attempt to provide all line data for future differential analyses, it was decided to fit the solar (and later stellar) line spectra with a single value for $\xi_{\mathrm{t}}$, whereas the external broadening usually combining rotation, macroturbulence and instrumental resolution in a convolved profile was allowed to vary with line strength or depth of line formation.

The micro- and macroturbulence velocities thus represent only an overall best fit attempt. In Tables 1 and 2 the HM model uses $\xi_{\mathrm{t}}=1.0 \mathrm{~km} \mathrm{~s}^{-1}$ and $\Xi_{\mathrm{rt}} \sim 2.5 \mathrm{~km} \mathrm{~s}^{-1}$, the TH model is best represented by $\xi_{\mathrm{t}}=0.85 \mathrm{~km} \mathrm{~s}^{-1}$ and $\Xi_{\mathrm{rt}} \sim 3.2 \mathrm{~km} \mathrm{~s}^{-1}$. Here, $\Xi_{\mathrm{rt}}$ refers to the radial-tangential macroturbulence model of Gray (1977). We note that, fortunately, the solar abundance results do not depend very much on our choice of $\xi_{\mathrm{t}}$ except for a few lines. The reason for this is that faint lines (subjected to high $\xi_{\mathrm{t}}$ values) do not depend very much on microturbulence at all, whereas strong lines (with very low $\xi_{\mathrm{t}}$ values) are defined mostly by van der Waals damping. The lines most sensitive to microturbulence thus emerge from similar atmospheric depths. A possible exception to this behaviour is encountered when fitting some of the Fe II lines (see Sect. 3.1). 
Table 2. 117 Fe I lines selected for solar NLTE analyses. f-values are from Nave et al.'s (1994) compilation. Abundances in the columns on the right refer to line syntheses based on the empirical solar model of Holweger \& Müller (1974, HM) and on a line-blanketed solar model assuming microturbulence velocities of $\xi_{\mathrm{t}}=1.0$ and $0.85 \mathrm{~km} \mathrm{~s}^{-1}$, respectively. $\log C_{6}$ refers to Anstee \& O'Mara $(1991,1995)$, where we have ignored the small differences in temperature dependence. Results are obtained from optimized profile fits with no allowance for individual variations of van der Waals damping constants. The HM results are for LTE, the other results for our final NLTE fits with $S_{\mathrm{H}}=0+, 0-, 1+, 5+$, and $5-$, where the abundance columns refer to the difference between atomic models with $(+)$ and without $(-)$ thermalization of levels above $7.3 \mathrm{eV}$ (see text). Remarks given in the last column are explained at the end of the table

\begin{tabular}{|c|c|c|c|c|c|c|c|c|c|c|c|c|c|}
\hline \multirow[t]{2}{*}{ Mult } & \multirow{2}{*}{\multicolumn{2}{|c|}{ Transition }} & \multirow[t]{2}{*}{$\lambda[\AA]$} & \multirow[t]{2}{*}{$E[\mathrm{eV}]$} & \multirow[t]{2}{*}{$\log g f$} & \multirow[t]{2}{*}{$\log C_{6}$} & \multicolumn{6}{|c|}{$\log \varepsilon_{\mathrm{Fe} \mathrm{I}}$} & \multirow[t]{2}{*}{ Rem } \\
\hline & & & & & & & HM & $0+$ & $0-$ & $1+$ & $5+$ & $5-$ & \\
\hline 1 & $a^{5} D_{4}$ & $-\mathrm{z}^{7} \mathrm{D}_{5}^{\mathrm{o}}$ & 5166.282 & 0.000 & -4.20 & -32.07 & 7.72 & 7.66 & 7.62 & 7.60 & 7.64 & 7.71 & $\mathrm{~b}$ \\
\hline 2 & $a^{5} D_{3}$ & $-\mathrm{z}^{7} \mathrm{~F}_{4}^{\mathrm{o}}$ & 4427.309 & 0.052 & -2.92 & -32.00 & 7.62 & 7.51 & 7.56 & 7.48 & 7.47 & 7.56 & $\mathrm{c}$ \\
\hline 15 & $a^{5} F_{2}$ & $-\mathrm{z}^{5} \mathrm{D}_{1}^{\mathrm{o}}$ & 5405.775 & 0.990 & -1.84 & -31.87 & 7.63 & 7.48 & 7.48 & 7.45 & 7.45 & 7.55 & $\mathrm{c}$ \\
\hline 15 & $\mathrm{a}^{5} \mathrm{~F}_{4}$ & $-\mathrm{z}^{5} \mathrm{D}_{4}^{\mathrm{o}}$ & 5397.775 & 0.915 & -1.99 & -31.88 & 7.62 & 7.45 & 7.44 & 7.42 & 7.43 & 7.53 & $\mathrm{c}$ \\
\hline 15 & $a^{5} F_{3}$ & $-\mathrm{z}^{5} \mathrm{D}_{2}^{\mathrm{o}}$ & 5371.489 & 0.958 & -1.65 & -31.87 & 7.56 & 7.44 & 7.48 & 7.43 & 7.43 & 7.53 & $\mathrm{c}$ \\
\hline 15 & $\mathrm{a}^{5} \mathrm{~F}_{4}$ & $-\mathrm{z}^{5} \mathrm{D}_{3}^{\mathrm{o}}$ & 5328.038 & 0.915 & -1.47 & -31.88 & 7.58 & 7.45 & 7.47 & 7.46 & 7.46 & 7.53 & $\mathrm{~b}, \mathrm{c}$ \\
\hline 15 & $a^{5} F_{5}$ & $-\mathrm{z}^{5} \mathrm{D}_{4}^{\mathrm{o}}$ & 5269.537 & 0.859 & -1.32 & -31.89 & 7.62 & 7.51 & 7.51 & 7.44 & 7.44 & 7.54 & $\mathrm{c}$ \\
\hline 36 & $\mathrm{a}^{3} \mathrm{~F}_{2}$ & $-\mathrm{z}^{3} \mathrm{~F}_{2}^{\mathrm{o}}$ & 5216.274 & 1.608 & -2.15 & -31.67 & 7.51 & 7.48 & 7.49 & 7.47 & 7.48 & 7.58 & \\
\hline 36 & $\mathrm{a}^{3} \mathrm{~F}_{3}$ & $-\mathrm{z}^{3} \mathrm{~F}_{3}^{\mathrm{o}}$ & 5194.941 & 1.557 & -2.09 & -31.68 & 7.57 & 7.46 & 7.48 & 7.46 & 7.45 & 7.55 & \\
\hline 41 & $\mathrm{a}^{3} \mathrm{~F}_{3}$ & $-\mathrm{z}^{5} \mathrm{G}_{4}^{\mathrm{o}}$ & 4404.750 & 1.557 & -0.14 & -31.56 & 7.57 & 7.44 & 7.51 & 7.45 & 7.45 & 7.53 & c \\
\hline 41 & $\mathrm{a}^{3} \mathrm{~F}_{4}$ & $-\mathrm{z}^{5} \mathrm{G}_{5}^{\mathrm{O}}$ & 4383.545 & 1.485 & 0.20 & -31.58 & 7.58 & 7.47 & 7.41 & 7.37 & 7.37 & 7.46 & c \\
\hline 42 & $\mathrm{a}^{3} \mathrm{~F}_{4}$ & $-\mathrm{z}^{3} \mathrm{G}_{5}^{\mathrm{o}}$ & 4271.760 & 1.485 & -0.16 & -31.55 & 7.55 & 7.45 & 7.46 & 7.42 & 7.42 & 7.49 & $\mathrm{~b}, \mathrm{c}$ \\
\hline 42 & $\mathrm{a}^{3} \mathrm{~F}_{4}$ & $-\mathrm{z}^{3} \mathrm{G}_{3}^{\mathrm{o}}$ & 4147.669 & 1.485 & -2.10 & -31.52 & 7.58 & 7.51 & 7.52 & 7.46 & 7.46 & 7.53 & \\
\hline 43 & $a^{3} F_{2}$ & $-\mathrm{y}^{3} \mathrm{~F}_{2}^{\mathrm{o}}$ & 4071.738 & 1.608 & -0.02 & -31.44 & 7.51 & 7.37 & 7.40 & 7.34 & 7.34 & 7.41 & c \\
\hline 43 & $\mathrm{a}^{3} \mathrm{~F}_{3}$ & $-y^{3} F_{3}^{\mathrm{o}}$ & 4063.594 & 1.557 & 0.06 & -31.47 & 7.51 & 7.38 & 7.38 & 7.39 & 7.39 & 7.44 & c \\
\hline 43 & $\mathrm{a}^{3} \mathrm{~F}_{4}$ & $-y^{3} F_{4}^{o}$ & 4045.812 & 1.485 & 0.28 & -31.49 & 7.51 & 7.38 & 7.39 & 7.38 & 7.38 & 7.45 & $\mathrm{c}$ \\
\hline 66 & $a^{5} \mathrm{P}_{2}$ & $-\mathrm{y}^{5} \mathrm{P}_{3}^{\mathrm{o}}$ & 5250.646 & 2.198 & -2.18 & -31.46 & 7.80 & 7.75 & 7.77 & 7.75 & 7.72 & 7.82 & \\
\hline 66 & $a^{5} \mathrm{P}_{1}$ & $-\mathrm{y}^{5} \mathrm{P}_{2}^{\mathrm{o}}$ & 5198.711 & 2.223 & -2.14 & -31.44 & 7.58 & 7.55 & 7.58 & 7.51 & 7.51 & 7.61 & \\
\hline 66 & $a^{5} P_{2}$ & $-\mathrm{y}^{5} \mathrm{P}_{1}^{\mathrm{o}}$ & 5079.223 & 2.198 & -2.07 & -31.43 & 7.65 & 7.62 & 7.66 & 7.59 & 7.58 & 7.68 & \\
\hline 68 & $a^{5} \mathrm{P}_{2}$ & $-\mathrm{x}^{5} \mathrm{D}_{3}^{\mathrm{o}}$ & 4494.563 & 2.198 & -1.14 & -31.30 & 7.59 & 7.53 & 7.55 & 7.51 & 7.49 & 7.59 & \\
\hline 68 & $a^{5} P_{1}$ & $-x^{5} S_{1}^{o}$ & 4447.717 & 2.176 & -1.34 & -31.27 & 7.81 & 7.70 & 7.58 & 7.63 & 7.62 & 7.72 & $\mathrm{~b}$ \\
\hline 71 & $a^{5} P_{3}$ & $-\mathrm{z}^{5} \mathrm{~S}_{2}^{\mathrm{o}}$ & 4282.402 & 2.176 & -0.78 & -31.24 & 7.29 & 7.23 & 7.24 & 7.17 & 7.18 & 7.26 & $\mathrm{c}$ \\
\hline 111 & $\mathrm{a}^{3} \mathrm{P}_{2}$ & $-\mathrm{z}^{3} \mathrm{P}_{2}^{\mathrm{o}}$ & 6421.350 & 2.279 & -2.03 & -31.56 & 7.59 & 7.53 & 7.53 & 7.53 & 7.57 & 7.67 & \\
\hline 114 & $\mathrm{a}^{3} \mathrm{P}_{1}$ & $-y^{3} D_{1}^{o}$ & 5141.739 & 2.424 & -2.24 & -31.35 & 7.66 & 7.61 & 7.64 & 7.57 & 7.57 & 7.66 & \\
\hline 114 & $\mathrm{a}^{3} \mathrm{P}_{2}$ & $-\mathrm{y}^{3} \mathrm{D}_{3}^{\mathrm{o}}$ & 5049.819 & 2.279 & -1.36 & -31.39 & 7.68 & 7.56 & 7.56 & 7.51 & 7.55 & 7.65 & \\
\hline 114 & $\mathrm{a}^{3} \mathrm{P}_{2}$ & $-\mathrm{y}^{3} \mathrm{D}_{2}^{\mathrm{o}}$ & 4924.769 & 2.279 & -2.11 & -31.37 & 7.61 & 7.59 & 7.61 & 7.54 & 7.56 & 7.66 & \\
\hline 152 & $\mathrm{z}^{7} \mathrm{D}_{5}^{\mathrm{o}}$ & $-\mathrm{e}^{7} \mathrm{D}_{5}$ & 4260.474 & 2.399 & 0.08 & -30.69 & 7.55 & 7.46 & 7.45 & 7.36 & 7.36 & 7.45 & $\mathrm{c}$ \\
\hline 152 & $\mathrm{z}^{7} \mathrm{D}_{2}^{\mathrm{o}}$ & $-\mathrm{e}^{7} \mathrm{D}_{3}$ & 4250.119 & 2.469 & -0.41 & -30.66 & 7.63 & 7.49 & 7.51 & 7.42 & 7.42 & 7.52 & $\mathrm{~b}, \mathrm{c}$ \\
\hline 152 & $\mathrm{z}^{7} \mathrm{D}_{1}^{\mathrm{o}}$ & $-\mathrm{e}^{7} \mathrm{D}_{2}$ & 4233.602 & 2.482 & -0.60 & -30.64 & 7.55 & 7.46 & 7.45 & 7.39 & 7.37 & 7.43 & \\
\hline 152 & $\mathrm{z}^{7} \mathrm{D}_{3}^{\mathrm{o}}$ & $-\mathrm{e}^{7} \mathrm{D}_{3}$ & 4222.213 & 2.449 & -0.97 & -30.65 & 7.55 & 7.48 & 7.48 & 7.41 & 7.41 & 7.48 & \\
\hline 152 & $\mathrm{z}^{7} \mathrm{D}_{1}^{\mathrm{o}}$ & $-\mathrm{e}^{7} \mathrm{D}_{1}$ & 4210.343 & 2.482 & -0.93 & -30.63 & 7.62 & 7.49 & 7.41 & 7.36 & 7.36 & 7.44 & $\mathrm{~b}$ \\
\hline 152 & $\mathrm{z}^{7} \mathrm{D}_{3}^{\mathrm{o}}$ & $-\mathrm{e}^{7} \mathrm{D}_{2}$ & 4187.039 & 2.449 & -0.55 & -30.64 & 7.51 & 7.47 & 7.47 & 7.41 & 7.40 & 7.50 & $\mathrm{~b}$ \\
\hline 168 & $\mathrm{a}^{3} \mathrm{H}_{5}$ & $-\mathrm{z}^{5} \mathrm{G}_{5}^{\mathrm{o}}$ & 6593.870 & 2.433 & -2.42 & -31.51 & 7.76 & 7.73 & 7.75 & 7.61 & 7.63 & 7.73 & \\
\hline 168 & $\mathrm{a}^{3} \mathrm{H}_{6}$ & $-\mathrm{z}^{5} \mathrm{G}_{5}^{\mathrm{O}}$ & 6494.980 & 2.404 & -1.27 & -31.52 & 7.62 & 7.53 & 7.55 & 7.50 & 7.50 & 7.60 & \\
\hline 168 & $\mathrm{a}^{3} \mathrm{H}_{5}$ & $-\mathrm{z}^{5} \mathrm{G}_{4}^{\mathrm{o}}$ & 6393.601 & 2.433 & -1.58 & -31.50 & 7.67 & 7.64 & 7.64 & 7.59 & 7.57 & 7.67 & \\
\hline 169 & $\mathrm{a}^{3} \mathrm{H}_{6}$ & $-\mathrm{z}^{3} \mathrm{G}_{5}^{\mathrm{o}}$ & 6252.555 & 2.404 & -1.69 & -31.49 & 7.66 & 7.58 & 7.56 & 7.55 & 7.55 & 7.65 & \\
\hline 169 & $\mathrm{a}^{3} \mathrm{H}_{5}$ & $-\mathrm{z}^{3} \mathrm{G}_{4}^{\mathrm{o}}$ & 6191.558 & 2.433 & -1.42 & -31.48 & 7.59 & 7.50 & 7.49 & 7.47 & 7.45 & 7.55 & \\
\hline 169 & $\mathrm{a}^{3} \mathrm{H}_{4}$ & $-\mathrm{z}^{3} \mathrm{G}_{3}^{\mathrm{o}}$ & 6136.615 & 2.453 & -1.40 & -31.47 & 7.62 & 7.53 & 7.54 & 7.50 & 7.49 & 7.59 & \\
\hline 207 & $\mathrm{~b}^{3} \mathrm{~F}_{3}$ & $-\mathrm{y}^{3} \mathrm{~F}_{4}^{\mathrm{o}}$ & 6322.685 & 2.588 & -2.43 & -31.43 & 7.74 & 7.70 & 7.73 & 7.65 & 7.65 & 7.74 & \\
\hline 207 & $\mathrm{~b}^{3} \mathrm{~F}_{4}$ & $-\mathrm{y}^{3} \mathrm{~F}_{4}^{\mathrm{o}}$ & 6230.723 & 2.559 & -1.28 & -31.44 & 7.72 & 7.57 & 7.66 & 7.64 & 7.64 & 7.74 & \\
\hline 207 & $\mathrm{~b}^{3} \mathrm{~F}_{2}$ & $-\mathrm{y}^{3} \mathrm{~F}_{3}^{\mathrm{o}}$ & 6200.312 & 2.608 & -2.44 & -31.42 & 7.69 & 7.67 & 7.73 & 7.63 & 7.63 & 7.73 & \\
\hline 207 & $\mathrm{~b}^{3} \mathrm{~F}_{3}$ & $-\mathrm{y}^{3} \mathrm{~F}_{3}^{\mathrm{o}}$ & 6137.691 & 2.588 & -1.40 & -31.42 & 7.66 & 7.56 & 7.58 & 7.59 & 7.59 & 7.69 & \\
\hline 207 & $\mathrm{~b}^{3} \mathrm{~F}_{2}$ & $-\mathrm{y}^{3} \mathrm{~F}_{2}^{\mathrm{o}}$ & 6065.482 & 2.608 & -1.53 & -31.40 & 7.64 & 7.55 & 7.58 & 7.56 & 7.57 & 7.65 & \\
\hline 268 & $\mathrm{a}^{3} \mathrm{G}_{5}$ & $-\mathrm{y}^{3} \mathrm{~F}_{4}^{\mathrm{o}}$ & 6677.987 & 2.692 & -1.42 & -31.42 & 7.66 & 7.57 & 7.59 & 7.60 & 7.59 & 7.67 & \\
\hline 268 & $\mathrm{a}^{3} \mathrm{G}_{4}$ & $-\mathrm{y}^{3} \mathrm{~F}_{3}^{\mathrm{o}}$ & 6592.913 & 2.727 & -1.47 & -31.40 & 7.51 & 7.48 & 7.52 & 7.50 & 7.50 & 7.60 & \\
\hline 268 & $\mathrm{a}^{3} \mathrm{G}_{3}$ & $-\mathrm{y}^{3} \mathrm{~F}_{2}^{\mathrm{o}}$ & 6546.239 & 2.758 & -1.54 & -31.39 & 7.54 & 7.48 & 7.51 & 7.47 & 7.47 & 7.57 & \\
\hline 318 & $\mathrm{z}^{7} \mathrm{~F}_{5}^{\mathrm{o}}$ & $-\mathrm{e}^{7} \mathrm{D}_{5}$ & 5006.119 & 2.832 & -0.62 & -30.70 & 7.51 & 7.46 & 7.46 & 7.42 & 7.42 & 7.50 & b \\
\hline 318 & $\mathrm{z}^{7} \mathrm{~F}_{6}^{\mathrm{o}}$ & $-\mathrm{e}^{7} \mathrm{D}_{5}$ & 4957.596 & 2.808 & 0.23 & -30.70 & 7.58 & 7.51 & 7.51 & 7.43 & 7.43 & 7.50 & c \\
\hline 318 & $\mathrm{z}^{7} \mathrm{~F}_{4}^{\mathrm{o}}$ & $-e^{7} D_{4}$ & 4957.298 & 2.851 & -0.41 & -30.69 & 7.61 & 7.56 & 7.55 & 7.51 & 7.51 & 7.59 & \\
\hline 318 & $\mathrm{z}^{7} \mathrm{~F}_{5}^{\mathrm{o}}$ & $-\mathrm{e}^{7} \mathrm{D}_{4}$ & 4920.503 & 2.832 & 0.07 & -30.68 & 7.61 & 7.51 & 7.53 & 7.49 & 7.49 & 7.57 & \\
\hline
\end{tabular}


Table 2. continued

\begin{tabular}{|c|c|c|c|c|c|c|c|c|c|c|c|c|c|}
\hline \multirow[t]{2}{*}{ Mult } & \multirow{2}{*}{\multicolumn{2}{|c|}{ Transition }} & \multirow[t]{2}{*}{$\lambda[\AA]$} & \multirow[t]{2}{*}{$E[\mathrm{eV}]$} & \multirow[t]{2}{*}{$\log g f$} & \multirow[t]{2}{*}{$\log C_{6}$} & \multicolumn{6}{|c|}{$\log \varepsilon_{\mathrm{Fe}}$} & \multirow[t]{2}{*}{ Rem } \\
\hline & & & & & & & HM & $0+$ & $0-$ & $1+$ & $5+$ & $5-$ & \\
\hline 318 & $\mathrm{z}^{7} \mathrm{~F}_{3}^{\mathrm{o}}$ & $-\mathrm{e}^{7} \mathrm{D}_{3}$ & 4918.994 & 2.865 & -0.34 & -30.66 & 7.58 & 7.50 & 7.47 & 7.41 & 7.41 & 7.47 & \\
\hline 318 & $\mathrm{z}^{7} \mathrm{~F}_{4}^{\mathrm{o}}$ & $-e^{7} D_{3}$ & 4891.492 & 2.851 & -0.11 & -30.66 & 7.57 & 7.51 & 7.49 & 7.42 & 7.42 & 7.49 & \\
\hline 318 & $\mathrm{z}^{7} \mathrm{~F}_{2}^{\mathrm{o}}$ & $-e^{7} D_{2}$ & 4890.755 & 2.875 & -0.39 & -30.65 & 7.58 & 7.52 & 7.50 & 7.43 & 7.43 & 7.51 & \\
\hline 383 & $\mathrm{z}^{7} \mathrm{P}_{2}^{\mathrm{o}}$ & $-e^{7} D_{3}$ & 5281.790 & 3.038 & -0.83 & -30.67 & 7.55 & 7.51 & 7.49 & 7.43 & 7.43 & 7.51 & $\mathrm{~b}$ \\
\hline 383 & $\mathrm{z}^{7} \mathrm{P}_{3}^{\mathrm{o}}$ & $-e^{7} D_{4}$ & 5266.555 & 2.998 & -0.39 & -30.68 & 7.57 & 7.51 & 7.51 & 7.45 & 7.45 & 7.53 & $\mathrm{~b}$ \\
\hline 383 & $\mathrm{z}^{7} \mathrm{P}_{4}^{\mathrm{o}}$ & $-\mathrm{e}^{7} \mathrm{D}_{5}$ & 5232.940 & 2.940 & -0.06 & -30.71 & 7.56 & 7.46 & 7.48 & 7.39 & 7.39 & 7.49 & $\mathrm{~b}, \mathrm{c}$ \\
\hline 383 & $\mathrm{z}^{7} \mathrm{P}_{2}^{\mathrm{o}}$ & $-e^{7} D_{1}$ & 5191.455 & 3.038 & -0.55 & -30.65 & 7.60 & 7.49 & 7.50 & 7.44 & 7.44 & 7.52 & $\mathrm{~b}, \mathrm{c}$ \\
\hline 383 & $\mathrm{z}^{7} \mathrm{P}_{4}^{\mathrm{o}}$ & $-e^{7} D_{4}$ & 5139.462 & 2.940 & -0.51 & -30.68 & 7.59 & 7.51 & 7.51 & 7.43 & 7.45 & 7.52 & \\
\hline 383 & $\mathrm{z}^{7} \mathrm{P}_{3}^{\mathrm{o}}$ & $-e^{7} D_{2}$ & 5139.251 & 2.998 & -0.74 & -30.65 & 7.57 & 7.50 & 7.51 & 7.40 & 7.40 & 7.47 & \\
\hline 383 & $\mathrm{z}^{7} \mathrm{P}_{4}^{\mathrm{o}}$ & $-e^{7} D_{3}$ & 5068.765 & 2.940 & -1.04 & -30.67 & 7.63 & 7.52 & 7.54 & 7.48 & 7.48 & 7.55 & $\mathrm{~b}$ \\
\hline 553 & $\mathrm{z}^{5} \mathrm{D}_{3}^{\mathrm{o}}$ & $-e^{5} \mathrm{D}_{4}$ & 5393.167 & 3.241 & -0.91 & -30.60 & 7.87 & 7.74 & 7.76 & 7.70 & 7.72 & 7.80 & \\
\hline 553 & $\mathrm{z}^{5} \mathrm{D}_{2}^{\mathrm{o}}$ & $-e^{5} D_{3}$ & 5339.929 & 3.266 & -0.72 & -30.56 & 7.74 & 7.61 & 7.65 & 7.59 & 7.59 & 7.69 & \\
\hline 553 & $\mathrm{z}^{5} \mathrm{D}_{4}^{\mathrm{o}}$ & $-e^{5} D_{4}$ & 5324.179 & 3.211 & -0.24 & -30.60 & 7.77 & 7.67 & 7.72 & 7.66 & 7.66 & 7.74 & \\
\hline 553 & $\mathrm{z}^{5} \mathrm{D}_{4}^{\mathrm{o}}$ & $-e^{5} D_{3}$ & 5217.389 & 3.211 & -1.16 & -30.56 & 7.73 & 7.63 & 7.67 & 7.61 & 7.61 & 7.69 & \\
\hline 554 & $\mathrm{z}^{5} \mathrm{D}_{4}^{\mathrm{o}}$ & $-\mathrm{e}^{5} \mathrm{~F}_{5}$ & 4736.773 & 3.211 & -0.75 & -30.33 & 7.65 & 7.51 & 7.50 & 7.49 & 7.49 & 7.57 & \\
\hline 686 & $\mathrm{z}^{5} \mathrm{~F}_{2}^{\mathrm{o}}$ & $-e^{5} D_{2}$ & 5624.542 & 3.417 & -0.90 & -30.53 & 7.77 & 7.70 & 7.73 & 7.71 & 7.69 & 7.77 & \\
\hline 686 & $\mathrm{z}^{5} \mathrm{~F}_{5}^{\mathrm{o}}$ & $-e^{5} D_{4}$ & 5615.643 & 3.332 & -0.14 & -30.59 & 7.79 & 7.69 & 7.74 & 7.68 & 7.68 & 7.75 & \\
\hline 686 & $\mathrm{z}^{5} \mathrm{~F}_{4}^{\mathrm{o}}$ & $-e^{5} D_{3}$ & 5586.755 & 3.368 & -0.14 & -30.56 & 7.66 & 7.58 & 7.58 & 7.56 & 7.56 & 7.64 & \\
\hline 686 & $\mathrm{z}^{5} \mathrm{~F}_{3}^{\mathrm{o}}$ & $-e^{5} D_{2}$ & 5572.842 & 3.396 & -0.31 & -30.53 & 7.64 & 7.51 & 7.54 & 7.51 & 7.51 & 7.61 & \\
\hline 686 & $\mathrm{z}^{5} \mathrm{~F}_{2}^{\mathrm{o}}$ & $-e^{5} D_{1}$ & 5569.618 & 3.417 & -0.54 & -30.51 & 7.64 & 7.53 & 7.59 & 7.54 & 7.54 & 7.64 & \\
\hline 816 & $\mathrm{z}^{5} \mathrm{P}_{2}^{\mathrm{o}}$ & $-e^{5} D_{3}$ & 6411.649 & 3.654 & -0.72 & -30.56 & 7.71 & 7.66 & 7.70 & 7.62 & 7.63 & 7.71 & $\mathrm{c}$ \\
\hline 816 & $\mathrm{z}^{5} \mathrm{P}_{3}^{\mathrm{o}}$ & $-e^{5} D_{4}$ & 6400.001 & 3.602 & -0.52 & -30.59 & 7.80 & 7.74 & 7.77 & 7.71 & 7.73 & 7.83 & \\
\hline 816 & $\mathrm{z}^{5} \mathrm{P}_{1}^{\mathrm{o}}$ & $-e^{5} D_{0}$ & 6302.493 & 3.686 & -0.91 & -30.51 & 7.39 & 7.28 & 7.36 & 7.26 & 7.28 & 7.37 & \\
\hline 816 & $\mathrm{z}^{5} \mathrm{P}_{3}^{\mathrm{o}}$ & $-e^{5} D_{3}$ & 6246.318 & 3.602 & -0.88 & -30.56 & 7.71 & 7.68 & 7.72 & 7.63 & 7.65 & 7.74 & $\mathrm{c}$ \\
\hline 816 & $\mathrm{z}^{5} \mathrm{P}_{2}^{\mathrm{o}}$ & $-e^{5} D_{1}$ & 6232.641 & 3.654 & -0.96 & -30.52 & 7.37 & 7.35 & 7.35 & 7.26 & 7.26 & 7.35 & \\
\hline 843 & $\mathrm{a}^{1} \mathrm{I}_{6}$ & $-\mathrm{z}^{1} \mathrm{H}_{5}^{\mathrm{o}}$ & 5242.491 & 3.634 & -0.97 & -30.63 & 7.50 & 7.47 & 7.48 & 7.41 & 7.43 & 7.51 & c \\
\hline 965 & $z^{3} F_{2}^{o}$ & $-e^{3} D_{1}$ & 5022.235 & 3.984 & -0.53 & -29.92 & 7.51 & 7.43 & 7.46 & 7.44 & 7.44 & 7.51 & $\mathrm{a}$ \\
\hline 965 & $\mathrm{z}^{3} \mathrm{~F}_{3}^{\mathrm{o}}$ & $-e^{3} D_{2}$ & 5014.942 & 3.943 & -0.30 & -29.93 & 7.47 & 7.36 & 7.38 & 7.36 & 7.36 & 7.46 & $\mathrm{a}, \mathrm{b}$ \\
\hline 965 & $\mathrm{z}^{3} \mathrm{~F}_{4}^{\mathrm{o}}$ & $-e^{3} D_{3}$ & 5001.863 & 3.881 & 0.01 & -29.56 & 7.32 & 7.21 & 7.24 & 7.21 & 7.21 & 7.29 & \\
\hline 984 & $\mathrm{z}^{3} \mathrm{D}_{2}^{\mathrm{o}}$ & $-e^{3} D_{2}$ & 4985.252 & 3.928 & -0.56 & -29.93 & 7.47 & 7.38 & 7.40 & 7.35 & 7.35 & 7.44 & $\mathrm{a}$ \\
\hline 1015 & $c^{3} \mathrm{~F}_{4}$ & $-\mathrm{w}^{3} \mathrm{~F}_{4}^{\mathrm{o}}$ & 6157.728 & 4.076 & -1.26 & -30.64 & 7.64 & 7.62 & 7.64 & 7.57 & 7.58 & 7.67 & $\mathrm{a}$ \\
\hline 1017 & $c^{3} F_{3}$ & $-\mathrm{y}^{3} \mathrm{H}_{4}^{\mathrm{o}}$ & 6127.906 & 4.143 & -1.40 & -30.57 & 7.59 & 7.58 & 7.55 & 7.51 & 7.51 & 7.61 & $\mathrm{a}$ \\
\hline 1018 & $c^{3} F_{4}$ & $-\mathrm{v}^{3} \mathrm{G}_{5}^{\mathrm{O}}$ & 6027.050 & 4.076 & -1.09 & -30.60 & 7.53 & 7.51 & 7.51 & 7.46 & 7.46 & 7.56 & $\mathrm{a}$ \\
\hline 1062 & $\mathrm{y}^{5} \mathrm{D}_{3}^{\mathrm{o}}$ & $-\mathrm{g}^{5} \mathrm{D}_{3}$ & 5473.900 & 4.154 & -0.76 & -29.91 & 7.49 & 7.46 & 7.47 & 7.42 & 7.42 & 7.51 & $\mathrm{a}$ \\
\hline 1077 & $\mathrm{y}^{5} \mathrm{~F}_{2}^{\mathrm{o}}$ & $-e^{5} F_{3}$ & 7568.899 & 4.283 & -0.60 & -30.28 & 7.28 & 7.25 & 7.30 & 7.25 & 7.27 & 7.36 & \\
\hline 1077 & $\mathrm{y}^{5} \mathrm{~F}_{5}^{\mathrm{o}}$ & $-\mathrm{e}^{5} \mathrm{~F}_{5}$ & 7511.020 & 4.178 & 0.10 & -30.35 & 7.56 & 7.40 & 7.45 & 7.45 & 7.46 & 7.54 & $\mathrm{~b}$ \\
\hline 1077 & $\mathrm{y}^{5} \mathrm{~F}_{1}^{\mathrm{o}}$ & $-e^{5} \mathrm{~F}_{2}$ & 7491.648 & 4.301 & -0.80 & -30.26 & 7.35 & 7.33 & 7.36 & 7.33 & 7.33 & 7.41 & \\
\hline 1087 & $\mathrm{y}^{5} \mathrm{~F}_{5}^{\mathrm{o}}$ & $-\mathrm{g}^{5} \mathrm{D}_{4}$ & 5662.516 & 4.178 & -0.57 & -29.92 & 7.57 & 7.46 & 7.46 & 7.45 & 7.45 & 7.52 & \\
\hline 1087 & $\mathrm{y}^{5} \mathrm{~F}_{4}^{\mathrm{o}}$ & $-\mathrm{g}^{5} \mathrm{D}_{3}$ & 5638.262 & 4.220 & -0.87 & -29.90 & 7.65 & 7.58 & 7.57 & 7.53 & 7.53 & 7.62 & $\mathrm{a}$ \\
\hline 1089 & $\mathrm{y}^{5} \mathrm{~F}_{5}^{\mathrm{o}}$ & $-\mathrm{g}^{5} \mathrm{~F}_{5}$ & 5162.272 & 4.178 & 0.02 & -30.26 & 7.67 & 7.54 & 7.59 & 7.59 & 7.59 & 7.67 & \\
\hline 1092 & $\mathrm{y}^{5} \mathrm{~F}_{5}^{\mathrm{o}}$ & $-\mathrm{f}^{5} \mathrm{G}_{6}$ & 5133.688 & 4.178 & 0.14 & -30.25 & 7.64 & 7.51 & 7.56 & 7.53 & 7.53 & 7.61 & \\
\hline 1094 & $\mathrm{y}^{5} \mathrm{~F}_{4}^{\mathrm{o}}$ & $-\mathrm{e}^{3} \mathrm{G}_{5}$ & 5074.748 & 4.220 & -0.20 & -30.17 & 7.65 & 7.52 & 7.61 & 7.58 & 7.58 & 7.66 & \\
\hline 1107 & $\mathrm{z}^{3} \mathrm{P}_{1}^{\mathrm{o}}$ & $-\mathrm{e}^{3} \mathrm{D}_{2}$ & 5753.122 & 4.260 & -0.69 & -29.90 & 7.50 & 7.41 & 7.45 & 7.45 & 7.45 & 7.57 & $\mathrm{a}$ \\
\hline 1144 & $\mathrm{z}^{5} \mathrm{G}_{4}^{\mathrm{O}}$ & $-\mathrm{h}^{5} \mathrm{D}_{3}$ & 5466.396 & 4.371 & -0.63 & -30.20 & 7.60 & 7.58 & 7.64 & 7.55 & 7.55 & 7.64 & \\
\hline 1145 & $\mathrm{z}^{5} \mathrm{G}_{2}^{\mathrm{O}}$ & $-\mathrm{f}^{5} \mathrm{G}_{2}$ & 5398.279 & 4.445 & -0.67 & -30.08 & 7.62 & 7.61 & 7.61 & 7.54 & 7.54 & 7.64 & \\
\hline 1145 & $\mathrm{z}^{5} \mathrm{G}_{3}^{\mathrm{O}}$ & $-\mathrm{f}^{5} \mathrm{G}_{3}$ & 5389.479 & 4.415 & -0.41 & -30.11 & 7.51 & 7.48 & 7.48 & 7.43 & 7.43 & 7.52 & \\
\hline 1146 & $\mathrm{z}^{5} \mathrm{G}_{6}^{\mathrm{O}}$ & $-\mathrm{e}^{5} \mathrm{H}_{7}$ & 5424.068 & 4.320 & 0.52 & -30.24 & 7.62 & 7.47 & 7.52 & 7.50 & 7.49 & 7.55 & \\
\hline 1146 & $\mathrm{z}^{5} \mathrm{G}_{5}^{\mathrm{O}}$ & $-e^{5} \mathrm{H}_{6}$ & 5383.369 & 4.312 & 0.64 & -30.23 & 7.38 & 7.26 & 7.29 & 7.23 & 7.23 & 7.31 & \\
\hline 1146 & $\mathrm{z}^{5} \mathrm{G}_{4}^{\mathrm{o}}$ & $-e^{5} H_{5}$ & 5369.961 & 4.371 & 0.54 & -30.16 & 7.36 & 7.25 & 7.30 & 7.29 & 7.27 & 7.35 & \\
\hline 1146 & $\mathrm{z}^{5} \mathrm{G}_{3}^{\mathrm{o}}$ & $-\mathrm{e}^{5} \mathrm{H}_{4}$ & 5367.466 & 4.415 & 0.44 & -30.10 & 7.37 & 7.26 & 7.29 & 7.26 & 7.25 & 7.32 & \\
\hline 1146 & $\mathrm{z}^{5} \mathrm{G}_{2}^{\mathrm{o}}$ & $-e^{5} H_{3}$ & 5364.871 & 4.445 & 0.23 & -30.06 & 7.48 & 7.38 & 7.40 & 7.39 & 7.39 & 7.45 & \\
\hline 1164 & $\mathrm{z}^{3} \mathrm{G}_{5}^{\mathrm{o}}$ & $-\mathrm{e}^{3} \mathrm{H}_{6}$ & 5415.199 & 4.386 & 0.64 & -30.16 & 7.38 & 7.27 & 7.29 & 7.27 & 7.27 & 7.35 & \\
\hline 1164 & $\mathrm{z}^{3} \mathrm{G}_{3}^{\mathrm{o}}$ & $-\mathrm{e}^{3} \mathrm{H}_{4}$ & 5410.909 & 4.473 & 0.40 & -30.05 & 7.33 & 7.25 & 7.28 & 7.30 & 7.27 & 7.34 & \\
\hline 1164 & $\mathrm{z}^{3} \mathrm{G}_{4}^{\mathrm{O}}$ & $-e^{3} H_{4}$ & 5321.108 & 4.434 & -0.95 & -30.05 & 7.30 & 7.28 & 7.28 & 7.20 & 7.20 & 7.29 & \\
\hline 1178 & $\mathrm{y}^{3} \mathrm{~F}_{4}^{\mathrm{o}}$ & $-\mathrm{f}^{5} \mathrm{G}_{5}$ & 6024.058 & 4.548 & -0.12 & -30.25 & 7.65 & 7.60 & 7.66 & 7.62 & 7.62 & 7.68 & \\
\hline 1180 & $\mathrm{y}^{3} \mathrm{~F}_{2}^{\mathrm{o}}$ & $-e^{3} G_{3}$ & 5930.179 & 4.652 & -0.23 & -30.09 & 7.56 & 7.54 & 7.56 & 7.50 & 7.50 & 7.57 & \\
\hline 1180 & $\mathrm{y}^{3} \mathrm{~F}_{4}^{\mathrm{o}}$ & $-\mathrm{e}^{3} \mathrm{G}_{4}$ & 5752.032 & 4.548 & -0.66 & -30.14 & 7.35 & 7.36 & 7.44 & 7.36 & 7.36 & 7.47 & \\
\hline
\end{tabular}


Table 2. continued

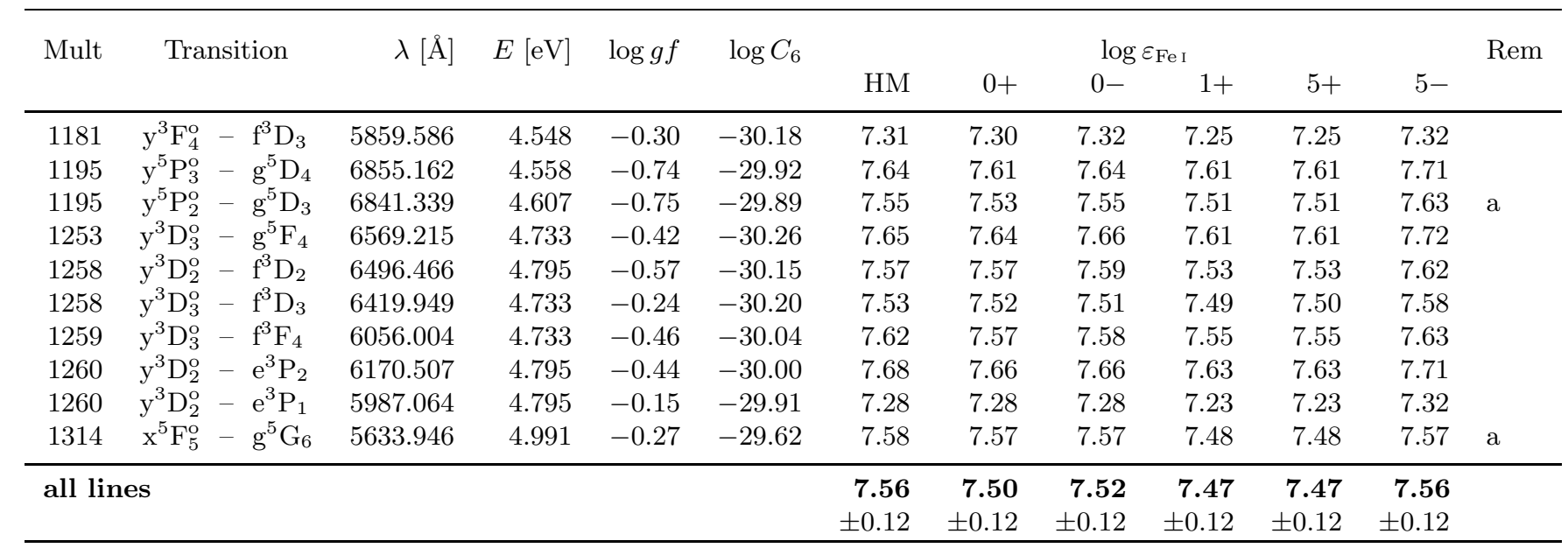

a) $\log C_{6}$ from Anstee \& O'Mara, extrapolated.

b) Line is blended or strongly asymmetric.

c) Bad core fit for NLTE model with $S_{\mathrm{H}}=0$.

\subsection{Fe II lines}

Comparing the different atomic models it has been shown that the excitation equilibrium of Fe II follows strictly thermal relations whereas the ionization itself predominantly affects Fe I. Consequently, Fe II lines are safely synthesized under the assumption of LTE. For stars other than the Sun they provide a basis of iron abundances that does not depend on the atomic model. As many Fe II lines as possible have been fitted to the solar flux spectrum to determine solar abundances. The results are given in Table 1 where for comparison we refer to recently published $f$-values of Kurucz (1992), Hannaford et al. (1992), Raassen \& Uylings (1998) and Schnabel et al. (1999). It is important to note that most of the Fe II flux line profiles fit to the mean microturbulence velocity of $\xi_{\mathrm{t}}=0.85 \mathrm{~km} \mathrm{~s}^{-1}$. An exception to that rule is multiplet 42 with $\lambda \lambda 4923,5018$ and $5169 \AA$ requiring $\xi_{\mathrm{t}}=1.1$ to $1.2 \mathrm{~km} \mathrm{~s}^{-1}$ to fit the line core width. All three lines apparently form in the upper photosphere with non-negligible contributions from chromospheric layers. Although differences seem to be only marginal they are systematic, and they show that Fe II 42 should be used only with care. The same holds for Fe II 73 , although $\lambda \lambda 7222,7224,7515$ and $7711 \AA$ emerge from relatively deep photospheric layers. We note that our original choice of Fe II lines included Mult $37 \lambda 4520 \AA$ and Mult $48 \lambda 5316 \AA$. Careful analyses with respect to other lines of these multiplets reveal that these two lines contribute only marginally to the observed solar lines, which therefore must be mainly due to unidentified blends.

The mean solar FeII abundances are presented in Table 1. Whereas the recent analysis of Schnabel et al. (1999), based on new lifetime measurements, arrives at $\log \varepsilon_{\mathrm{Fe} \text { II }, \odot}=7.42 \pm 0.09$, work by Raassen \& Uylings (1998) seems to indicate a significant systematic shift of their calculated $f$-values with respect to both $f$-values derived from lifetime measurements and those calculated by Kurucz (1992). The FerRum project (Sikström et al. 1999) is currently trying to resolve the reason for the discrepancy of the atomic data. Our results are transformed into solar Fe II abundances only with due recognition of the inhomogeneous set of $f$-values found in the literature. Using the 8 lines in common with Schnabel et al. (1999) we obtain for the HM atmosphere $\log \varepsilon_{\mathrm{Fe} \text { II }, \odot}=7.47 \pm 0.09$; our slightly increased value results not only from the restricted choice of lines, but also depends on the fact that we have used flux profile fits. Using only Kurucz (1992) $f$-values, the result for 17 lines is $\log \varepsilon_{\mathrm{Fe} \mathrm{II}, \odot}=7.45 \pm 0.09$. Finally, the Raassen \& Uylings (1998) $f$-values of 25 lines give $\log \varepsilon_{\mathrm{Fe} \text { II }, \odot}=7.56 \pm 0.06$. In view of the unresolved discrepancies of the $f$-values the resulting abundance differences are not surprising.

Results for the TH solar model atmosphere do not differ very much from those for the HM model. $\log \varepsilon_{\mathrm{Fe} \text { II }, \odot}=$ $7.46 \pm 0.09$ is the solar FeII abundance when using the Schnabel et al. $f$-values, Kurucz's $f$-values give $\log \varepsilon_{\mathrm{Fe} \text { II } \odot \odot}=7.44 \pm 0.10$, and for the Raassen \& Uylings $f$-values we obtain $\log \varepsilon_{\mathrm{Fe} \text { II }, \odot}=7.56 \pm 0.07$. If Mult 42 were calculated instead with an enhanced chromospheric microturbulence contribution, $\xi_{\mathrm{t}}=1.2 \mathrm{~km} \mathrm{~s}^{-1}$, the abundance mean would be lowered by $0.01 \ldots 0.02$, depending on the total number of lines involved.

The large rms abundance scatter found for both atmospheric models in Fig. 8 are disturbing. They are significantly higher than expected on the basis of the excellent solar line spectra. Whereas part of the scatter is definitely the result of representing granular motions by a constant microturbulence and paying only restricted attention to van der Waals broadening, a dominant source of errors still seem to be the $f$-values, whether they are experimental or calculated. Internal comparison of the abundances found in common multiplets also seems to indicate that there exist single lines that apparently require high 
abundances. Examples are $\lambda 4491 \AA$ in Mult $37, \lambda 5018 \AA$ in Mult $42, \lambda 5362 \AA$ in Mult 48 , and $\lambda 6416 \AA$ in Mult 74 . As in the case of $\lambda 5284 \AA$ (Mult 41), it is tempting to speculate that these lines carry unknown blends. Lines of multiplets 27 to 38 arising from the $\mathrm{b}^{4} \mathrm{P}$ and $\mathrm{b}^{4} \mathrm{~F}$ levels result in excessively low abundances. This does not seem to depend on the source of the $f$-values as it does for the other terms. At present this trend with excitation energies remains unexplained although there is a marginal dependence of the Fe II abundances on the damping factor $\Delta \log C_{6}$.

\subsection{The role of hydrogen collisions}

Before discussing the individual lines of $\mathrm{Fe} I$ it is necessary to recall that NLTE line formation depends critically on the proper choice of the hydrogen collision enhancement factor $S_{\mathrm{H}}$ (see Sect. 2.3). This has also been briefly discussed by both Thévenin \& Idiart (1999) and Gratton et al. (1999). Figure 9 shows profile fits for selected $\mathrm{Fe}$ I lines with different values of $S_{\mathrm{H}}$. The lines are selected to represent fair coverage of excitation energies. Deviations from LTE in the solar atmosphere are small as expected, and profile fit deficiencies could be removed by individual adjustment of damping constants in some cases. Differences between $S_{\mathrm{H}}=0+$ and $S_{\mathrm{H}}=5+$ are surprisingly small for most of the excited lines, but they are most evident for the strong low-excitation lines. Changes of the core profiles are not monotonous in a number of lines as shown in Fig. 9. What is called "profile fits" here refers to a qualitative interactive comparison of observed and theoretical line profiles with no attempt to quantify the "optimized fit". This seems adequate because we have artificially limited the free parameter space specifying in advance data like microturbulence and damping (in the case of FeI).

The reaction of the statistical model in response to changing the hydrogen collision rates should be very similar to that of $\mathrm{Mg}$ I (Zhao et al. 1998) or Al I (Baumüller \& Gehren 1996). The strong photoionization cross-sections of these ions are compensated by varying the collision efficiency with excitation energy. Therefore, similarly to $\mathrm{Mg}$ I a series of synthetic NLTE profiles has been calculated for Fe I with a hydrogen collision enhancement factor $S_{\mathrm{H}}=2500 \exp \left(n E_{n}\right)$, where $n$ is the effective quantum number and $E_{n}$ the upper level excitation energy in $\mathrm{eV}$. This approximation leads to a reasonable fit in the cores of the strong low-excitation lines. For Fe I in general it is, however, not the most convincing approach since it fails to fit the cores of many lines at intermediate and higher excitation energies (see the profiles labeled "exp" in Fig. 9).

Consequently, the most convincing choice of the hydrogen collision parameter $S_{\mathrm{H}}$ is either a value of 0 (no hydrogen collisions at all) or 5 . If hydrogen collisions are neglected there are only marginal differences between the models with or without thermalization of the highly excited terms $(0+$ and $0-$, see Table 2$)$. However, strong hydrogen collisions depend significantly on the coupling of these terms with the Fe II ground state. Besides the differences of abundances necessary to fit the line profiles it is important to note that also the line cores are slightly better reproduced with the $5+$ model. Even careful inspection of the line profiles does not allow a final decision whether hydrogen collisions are necessary or not. The fact that all strong line cores synthesized with $S_{\mathrm{H}}=0$ do not fit the solar observations must not necessarily rule out this choice. An explanation for the strong line cores is given in Fig. 10 which shows the observed Fe I line centre residual solar flux as a function of wavelength. The decrease of fit quality is represented by filled circles of decreasing size and darker grey color. It is evident that the majority of all lines is well fitted with $S_{\mathrm{H}}=0$ (NLTE) calculations, shown also in Fig. 9. A small fraction of the lines shows similar fits for all models, whereas lines with extremely deep cores are reproduced better by calculations with $S_{\mathrm{H}}=5$ or even LTE (see Fig. 9). This seems to be at variance with the notion that the strongest line cores are radiated from the highest photospheric layers where, due to radiative losses and decreasing collision rates NLTE effects should be strongest. The dashed curve in Fig. 10 is a pseudo-saturation curve calculated from $B_{\lambda}(4200 \mathrm{~K}) / B_{\lambda}(6000 \mathrm{~K})$. It approximates the emission of continuum photons $(6000 \mathrm{~K})$ and that of photons emitted near the temperature minimum (4200 K) where the cores of the stronger lines are formed. This suggests that residual core fluxes found below this curve indicate substantial contributions to the line core arising from the solar chromosphere, which is not part of our atmospheric models. In fact, all bad fits of solar line cores are due to lines with extremely low residual fluxes. Lines arising from purely photospheric layers are all well represented by $S_{\mathrm{H}}=0$ (but also by $S_{\mathrm{H}}=5$ ).

Thévenin \& Idiart (1999) mention that their Fe equivalent widths do not depend very much on whether $S_{\mathrm{H}}=0$ or 1. This is in fact true for most of the solar lines as long as only equivalent widths are examined. As shown in many excited Fe I lines (see also Fig. 9) it is not true even for $W_{\lambda}$ if weaker lines are analyzed. Many solar lines display the failure when fitting equivalent widths instead of line profiles; part of the $\log C_{6}$ enhancement always required to fit solar $W_{\lambda, \mathrm{FeI}}$ is an artefact resulting from the bad line core fits that in turn require stronger line wings to compensate for a missing fraction of equivalent width. However, Fe I lines in the Sun and in metal-poor stars are extremely different in equivalent width, and the proper choice of neutral hydrogen collision rates is therefore very important. It is even more important in extremely metal-poor stars where electron collisions play a minor role due to missing electron-donators, whereas simultaneously the UV radiation field is strongly increased as a result of reduced metal-line blocking. Gratton et al. (1999) calibrated their hydrogen collisions with a factor $x$ which they determined from RR Lyrae stars. They adopt $\log x=1.5$ which is an order of magnitude greater than both the scaling factor assumed by Thévenin \& Idiart and our solar choice with $0 \leq S_{\mathrm{H}} \leq 5$. We will therefore extend the test of 

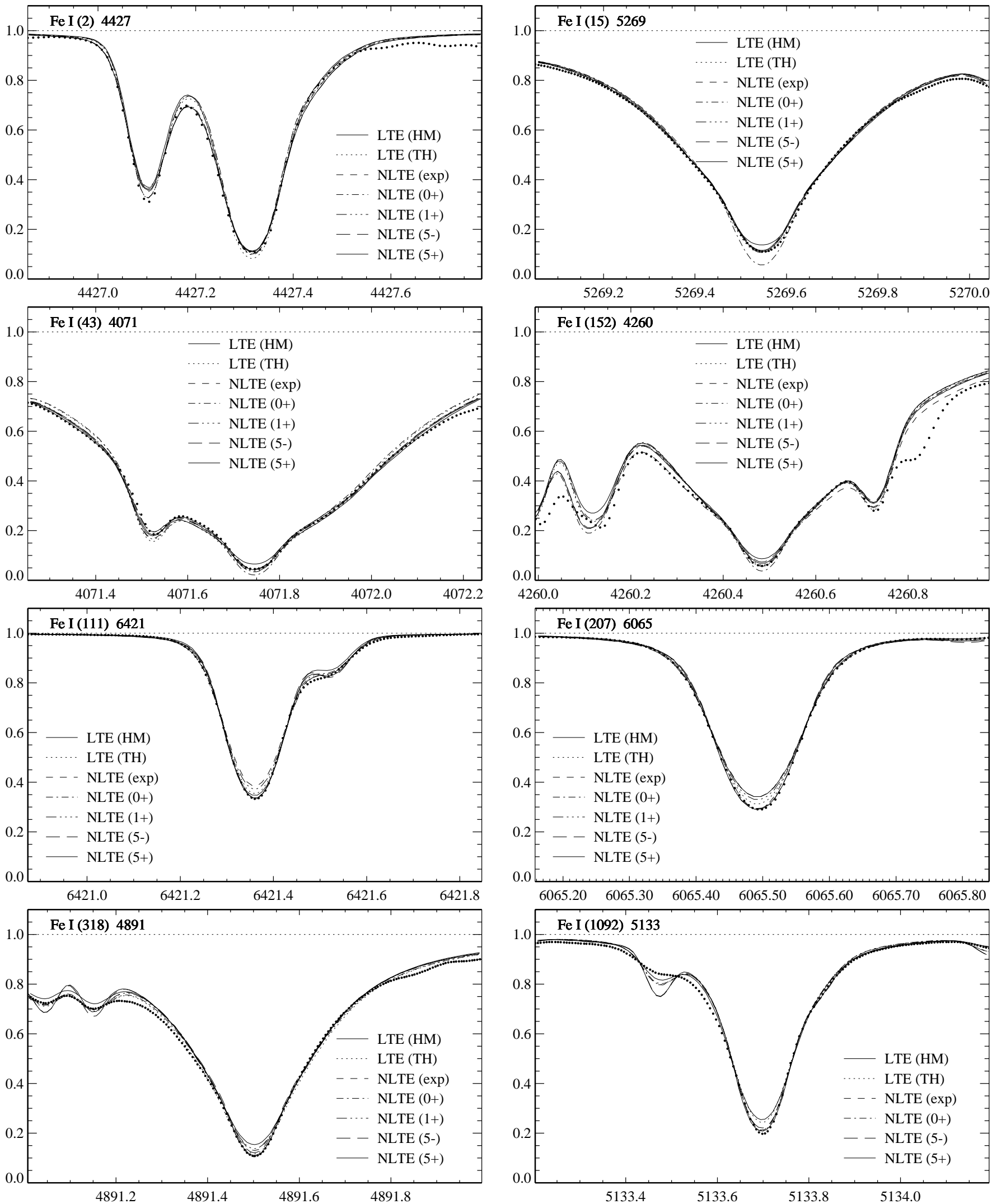

Fig. 9. Sample of Fe I lines in the solar spectrum of Kurucz et al. (1984). Observed spectra are indicated by small filled circles. Different synthetic spectra are labeled with numbers in parentheses indicating $S_{\mathrm{H}}$ and thermalization for levels above $7.3 \mathrm{eV}(+)$. "exp" refers to exponentially scaled $S_{\mathrm{H}}$ (see text). Upper two rows show lines with well saturated cores; without individual fit of either hydrogen collisions or depth-dependent (chromospheric) microturbulence velocities NLTE calculations with $S_{\mathrm{H}}<1$ produce line cores that are too deep. Bottom four panels represent the majority of solar photospheric Fe I lines which fit best to synthetic profiles calculated with either $S_{\mathrm{H}}=0+$ or $S_{\mathrm{H}}=5+$ 


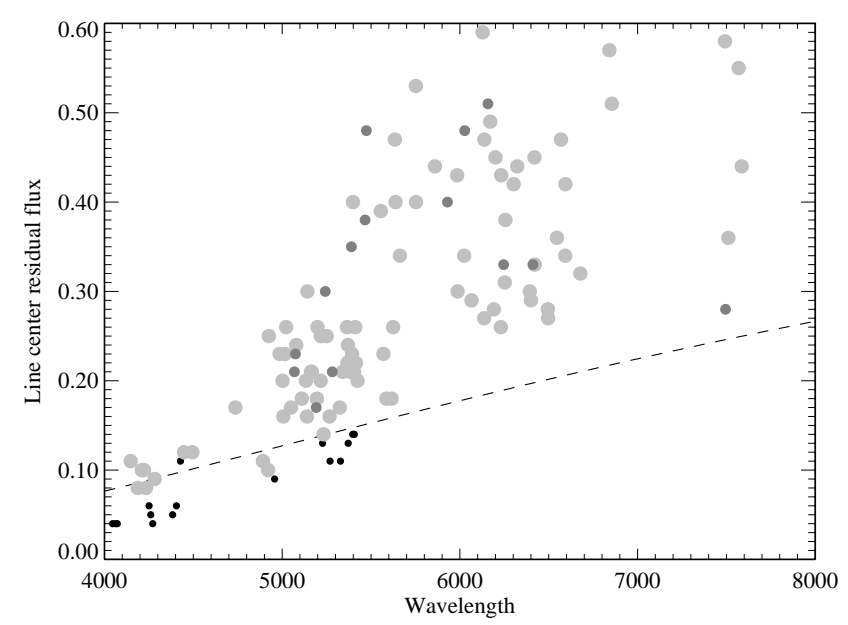

Fig. 10. Observed solar line centre residual flux shown as function of wavelength (in $\AA$ ). Large circles filled with light grey colour are lines for which NLTE calculations with $S_{\mathrm{H}}=0$ produce the best fit (see Fig. 9). Smaller circles filled in dark grey refer to fits where NLTE with $S_{\mathrm{H}}=0$ is rivalled by LTE calculations or NLTE with other values of $S_{\mathrm{H}}$. Black dots stand for bad line core fits with $S_{\mathrm{H}}=0$ (see Fig. 9), the NLTE profiles being too deep. Below the dashed curve substantial contributions are supposed to arise from the solar chromosphere

the Drawin formula to metal-poor stars in a forthcoming paper.

\subsection{Line profiles and abundances}

The selected sample of solar Fe I lines presented in Table 2 is used here to discriminate LTE profiles and different NLTE profiles, i.e. from atomic models with different hydrogen collision factors $S_{\mathrm{H}}$ or different treatment of highly excited levels. All lines have been synthesized from between 2 to 10 different models with LTE in both HM and TH, and with NLTE using a number of different approximations to the hydrogen collisions. Neither a variation of electron collisions within a factor of 10 nor of the representation of the Fe atom (i.e. the number of levels in Fe II) produced a significant change in the synthetic profiles. At this stage of refinement it is impossible to use the data to derive very accurate solar iron abundances. Taken at face value, the resulting abundance values are only reflections of the input of sometimes unreliable oscillator strengths. We note that we have not tried to improve these data since our aim is to produce $\log g f \varepsilon_{\odot}$ values for differential analyses of other stars. The $f$-values compiled by Nave et al. (1994) are from different sources, and their reliability must be questioned in view of abundances found for some lines in Mult 1077, 1146 or 1260. Problems with highly excited lines in particular appear in both LTE and NLTE profiles, and in both empirical and theoretical solar models. Therefore they cannot be the result of our special thermalization of levels above $7.3 \mathrm{eV}$. Whenever large discrepancies with respect to the meteoritic iron abundance occurred, the profile fits show a tendency for $\log C_{6}$ to deviate from the calculated data. Such lines will have to be reanalyzed with improved oscillator strengths, and they will be excluded from application in metal-poor stars.

It turns out that in most of the lines the necessary corrections to van der Waals damping are far smaller than expected. The calculations of Anstee \& O'Mara (1991, 1995) are in fact significantly more realistic than any other approximation used so far for Fe I. This is the reason that we have used their results without modification although we are aware that individual lines or multiplets may have to be revised in terms of improved damping constants. To a certain degree the $f$-values and the $\log C_{6}$ can be exchanged in their influence on the line profiles. However, multiplets with a range of line strengths make it possible to disentangle the respective influence of the two sources of line broadening. This has been checked with Mult 15, 41, 168 , and 318 , and the profile analysis confirms the notion that our failure to fit the cores of the very strong lines with $S_{\mathrm{H}}=0$ is not the result of badly chosen damping parameters.

The data in Table 2 together with Fig. 7 suggest that the enhancement of the $C_{6}$ values by typical factors of $\approx 10$ used in HM model atmosphere abundance analyses must be considered as a compensation of unrealistic damping constant approximations (i.e. Unsöld's formula) for most of the lines investigated here. While such enhancement factors are usually required for equivalent width analysis, the corresponding results of Anstee \& O'Mara are recovered here in most cases for the HM model even with profile analysis, simply because the majority of the lines in Table 2 is so strong that the cores do not contribute very much to $W_{\lambda}$. It is, however, the line cores that are often not fitted by LTE profiles using the HM atmosphere (see Fig. 9). Table 2 entries average on $\log \varepsilon_{\mathrm{Fe} \text { I,HM }}=7.56 \pm 0.12$ and $\log \varepsilon_{\mathrm{Fe} \text { I,TH }}=7.47 \ldots 7.56 \pm 0.12$. The excessive scatter for both models comes from a few multiplets for which the Nave et al. (1994) oscillator strengths seem to be systematically offset. This is particularly evident for Mult 168 with $\overline{\Delta \log \varepsilon} \sim 0.12$, for Mult 1077 where $\overline{\Delta \log \varepsilon} \sim-0.16$, for Mult 1146 with $\overline{\Delta \log \varepsilon}=-0.12$, and for Mult 1164 with $\overline{\Delta \log \varepsilon} \sim-0.22$, where we have used the notation $\overline{\Delta \log \varepsilon}$ to describe the deviation of the mean multiplet abundance from the mean of all lines. A number of original members of our line sample was removed at this stage of analysis because of clear evidence for a strong line blend. These removals do not affect the resulting abundances very much, but they reduce the scatter.

Table 2 presents individual abundances obtained from profile fits, where no freedom was left for adjustment of $\xi_{\mathrm{t}}$ and $\log C_{6}$. Figure 11 documents that the strong scatter currently makes the detection of any abundance trend with excitation energy impossible for the Sun; of course, this would not be true for other stars if analyses were strictly differential, i.e. using not absolute but solar $f$-values. It also shows that while zero hydrogen collisions give the best fits to most of the line cores, those with relatively strong collisions $\left(S_{\mathrm{H}}=5\right)$ depend on the treatment 


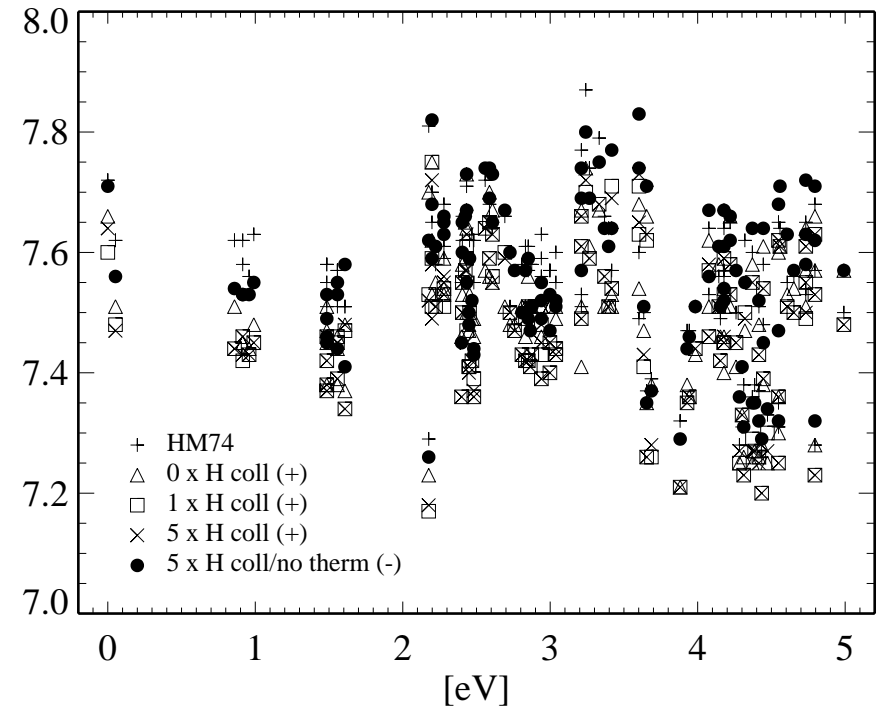

Fig. 11. Solar Fe I abundances as a function of lower level excitation energy calculated from profile fits for different hydrogen collision rates (see text)

of the highly excited levels. It is interesting to note that strong hydrogen collisions applied to the standard model atom (i.e. without forcing the highly excited levels into LTE with respect to Fe II) in our theoretical atmospheric model produce nearly the same Fe I abundances as LTE in the empirical HM model. However, the result that both the HM model under LTE conditions and the TH model with NLTE calculations produce similar abundances, if profile fits are preferred to equivalent width analysis, is not surprising. It shows nicely how the temperature stratification of the HM model can be used to compensate the NLTE populations of the Fe I levels.

\section{Discussion}

The complexity of the results and their dependence on a number of considerations that are not easily supported by basic data may leave the unprepared reader somewhat in confusion. While it is evident that in principle photoionization-dominated neutral elements such as Fe I should be more prone to NLTE, our investigation does not unequivocally present the true amount of such effects. The fact that in the Sun under certain assumptions no hydrogen collisions lead to very much the same mean Fe I abundance as those with $S_{\mathrm{H}}=5$ (including thermalization of upper levels) is both surprising because this was not expected, and it is annoying because it does not allow a unique solution based only on the solar strong line spectrum.

\subsection{Comparison with previous analyses}

We have already mentioned above some details in which our atomic models differ from those of Thévenin \& Idiart (1999) and of Gratton et al. (1999). At a first glance it seems that both groups have used relatively strong hy- drogen collisions although based on different considerations. While this only marginally affects the solar equivalent widths (see above), it is necessary to be aware of a significantly different influence on the spectra of metalpoor stars in which only those Fe I lines are detected that are very strong in the Sun. In such cases substantial differences may arise between analyses based on NLTE populations calculated with or without hydrogen collisions. The different level populations of Fe I are displayed in Fig. 4 of Thévenin \& Idiart and in Fig. 5 of Gratton et al.

The departure coefficients of Thévenin \& Idiart, however, show some similarity with our results for $S_{\mathrm{H}}=0$ (see our Figs. 3 and 6). This confirms that the results of Thévenin \& Idiart are in fact obtained with zero hydrogen collisions (Thévenin, priv. comm.), because even with $S_{\mathrm{H}}$ as small as 0.01 the Fe I levels with low excitation energies should be tied together very closely as is demonstrated in our Figs. 6a and d. Note that in metal-poor stars using $S_{\mathrm{H}}=0$ instead of $S_{\mathrm{H}}=5$ may produce abundance differences of more than 0.2 dex.

The solar Fe I populations of Gratton et al. are completely different. This must be understood as the consequence of their highly incomplete description of the atomic structure, and of their exceedingly strong hydrogen collisions corresponding to $S_{\mathrm{H}} \approx 30$. Thus their hydrogen collisions dominate the statistical equilibrium both in the Sun and in metal-poor stars. Another difference is at least as important, and that is the lack of adequate photoionization cross-sections in the work of Gratton et al. Their approximations seem to underestimate the true Fe I photoionization rates by large amounts. Combining the inefficiency of hydrogenic photoionization with the dominating influence of hydrogen collisions produces nearly thermal level populations throughout the photospheres of cool stars of any type, so that it is easy to understand that they do not obtain large deviations from LTE.

We mention that a comparison with previous work of Steenbock (1985) and Gehren et al. (1991) is problematic in that their results were based on a differential calculation of equivalent widths. As explained above this could lead to different results as compared with line profile analysis since mostly weak or medium-strong lines were employed.

The results of our analysis of the solar line spectrum demonstrate once again that the Sun is one of the best laboratories to determine $f$-values once the photospheric Fe I abundance has been specified, say, as being identical to the meteoritic value. Some of the discrepancies displayed in Table 2 are definitely the result of faulty measurements in terrestrial laboratories. The plane-parallel horizontally homogeneous atmospheric model of the Sun - whether empirical or line-blanketed - is presumably the best iron oven available. Yet some lines are possibly affected by undetected blends, and in such cases solar $f$-values cannot be determined. 


\subsection{Solar ionization equilibrium}

The FeII/Fe I ionization equilibrium obtained from Tables 1 and 2 is not conclusive because the abundances are not reliable. For Fe II this is best seen in the significant discrepancy between lines with multiplet numbers below and above 40. The distinct abundance "step" amounts to $\log \varepsilon_{\mathrm{Fe} \text { II } \odot \odot} \sim 0.12$. For Fe I the excessive scatter displayed in Fig. 2 does not allow a precise determination of the mean abundance. With the present data our NLTE model labeled "5-" (see Table 2) reproduces a perfect ionization equilibrium for the TH model as does the LTE abundance analysis for the HM model, both resulting in a common solar Fe abundance of $\log \varepsilon_{\mathrm{Fe}, \odot}=7.56 \pm 0.11$, which is reasonably close to the meteoritic value. However, the NLTE models with $S_{\mathrm{H}}<5$ or with highly excited $\left(E_{\mathrm{l}}>7.3 \mathrm{eV}\right)$ levels forced into LTE with respect to Fe II cannot be ruled out on the basis of the ionization equilibrium alone.

We note here that we have not attempted to include a list of weak lines in our analysis. Solar abundance analyses are normally restricted to such lines because they are not affected by microturbulence or damping. A corresponding extension of our analysis could provide evidence against some of the present atomic model assumptions. Such work will be followed in a separate investigation in which we will come back to the problem of the solar iron abundances.

\subsection{Conclusions}

The best overall fits to the observed solar flux profiles in Fig. 9 are produced by the atomic models with strong hydrogen collisions $(5+$ and $5-)$. As shown in Figs. 5 and 6 the case $5-$ provides notable departures from LTE of the level populations but only marginal deviations of the line source functions from the Planck function in the solar photosphere. Due to the corresponding shift of the line-forming regions towards deeper layers the synthesized line wings are slightly shallower and thus require enhanced iron abundances with respect to the $5+$ model to reproduce the observed profiles. The nearly thermalized line source functions are a common feature of strong $b b$ collisional coupling introduced here, whereas photoionization dominates the level populations.

Together, Figs. 9 and 10 also support the atomic model with no hydrogen collisions $(0+)$, which may be considered an extreme NLTE case. This is an unexpected result, and it would be strongly at variance with previous publications if applied to other cool stars, such as the extremely metal-poor subgiant HD 140283, for which our $S_{\mathrm{H}}=0$ model would produce an ionization equilibrium with an abundance gap of $\log \varepsilon_{\mathrm{Fe} \text { II }}-\log \varepsilon_{\mathrm{Fe} \text { I }} \approx 0.35$. Fortunately, all models with hydrogen collision factors $S_{\mathrm{H}}$ between 0.01 and 2 show inferior profile fits.

Close inspection of the ionization equilibrium (Tables 1 and 2) would lead us to prefer the minimum NLTE case with hydrogen collisions enhanced by a factor $S_{\mathrm{H}}=5$ and no attempt to thermalize the upper Fe I levels. The large scatter of iron abundances as determined from fitting the lines of Tables 1 and 2 is, however, a strong argument against selecting the atomic model on the grounds of the Fe II/Fe I ionization equilibrium. Although it is somewhat surprising that both the LTE model of Holweger \& Müller (1974) and the theoretical NLTE model with $S_{\mathrm{H}}=5-$ give the same Fe I abundances, and though this Fe I abundance is in perfect agreement with that of Fe II, it should be emphasized that virtually all models, whether LTE or NLTE lead to acceptable solar ionization equilibria within their $1 \sigma$ error bars. Additionally, the model with $S_{\mathrm{H}}=5+$ produces the best line core fits while the Fe I abundance is lower by roughly 0.1 dex. The low Fe I abundance would then fit better to a correspondingly lower Fe II abundance resulting from experimental $f$-values.

It remains to conclude that the proper atomic model, and in particular the influence of neutral hydrogen collisions can not be chosen from comparison with the strong line solar flux spectrum alone. It will be necessary to confirm the present results using a selected sample of weak solar lines that do not depend on damping (and not much on microturbulence either). As an independent test it is important to examine similar spectra of a number of reference stars, for which the surface gravity and thus the iron ionization equilibrium is determined by HipPARCOS parallaxes and/or the wings of the $\mathrm{Mg} \mathrm{I} b$ lines.

As compared with the previous analyses of Thévenin \& Idiart (1999) or Gratton et al. (1999) our results are different because of a different choice of interaction cross-sections. The impact of Bautista's (1997) bound-free absorption on the iron ionization equilibrium is more important for metal-poor stars than it is for the Sun, and it remains to be explained why departures from LTE are very moderate even for the most extreme halo stars. Basically, the problem is always traced back to the solar spectrum, its description by a simplified 1D atmospheric model, and an incoherent set of laboratory $f$-values. The problem of the $f$-values becomes particularly important noting that the mean abundances derived from single sources of Nave et al.'s compilation are often more different than mean abundances obtained for the different atomic models. This will be more easily demonstrated with a set of weak Fe I lines.

The validity of our rigid assumptions concerning linebroadening (i.e. fixing both microturbulence and damping parameters) is to be judged only as a first attempt. We have repeatedly noticed that a depth-dependent microturbulence velocity would represent the granular flow much better than a single number. Such a stratification would also be tied to large-scale motions hidden in our macroturbulence parameter. The introduction of convective overshooting as advocated by Castelli et al. (1997) or Kupka (1999) presents yet another source of uncertainty. Therefore, the most reliable result is the NLTE abundance difference with respect to LTE in the same atmospheric model.

Acknowledgements. We thank M. A. Bautista for making available his bf Fe I photoionization cross-sections. Part of this 
work was funded by the Deutsche Forschungsgemeinschaft (DFG) under grants Ge 490/12-1 and -2, and by the German ministry of Science and Technology (BMBF) under grant 05 2MU114(7). JS acknowledges support from the Chinese Academy of Science, and LM appreciates a travel grant from the Max-Planck-Gesellschaft.

\section{References}

Allen, C. W. 1973, Astrophysical quantities, 3rd ed. (Athlone Press, London)

Allende Prieto, C., García López, R., Lambert, D., \& Gustafsson, B. 1999, ApJ, 527, 879

Anstee, S. D., \& O'Mara, B. J. 1991, MNRAS, 253, 549

Anstee, S. D., \& O'Mara, B. J. 1995, MNRAS, 276, 859

Asplund, M., Nordlund, Å., Trampedach, R., \& Stein, R. F. 1999, A\&AL, 346, 17

Athay, R. G., \& Canfield, R. C. 1969, ApJ, 156, 695

Athay, R. G., \& Lites, B. W. 1972, ApJ, 176, 809

Barklem, P. S., Piskunov, N., \& O'Mara, B. J. 2000, A\&A, $355, \mathrm{~L} 5$

Baumüller, D., \& Gehren, T. 1996, A\&A, 307, 961

Baumüller, D., \& Gehren, T. 1997, A\&A, 325, 1088

Baumüller, D., Butler, K., \& Gehren, T. 1998, A\&A, 338, 637

Bautista, M. A. 1997, A\&AS, 122, 167

Bikmaev, I. F., Bobritskij, S. S., \& Sakhibullin, N. A. 1990, Sov. Astron. J. Lett., 16, 213

Blackwell, D. E., Lynas-Gray, A. E., \& Smith, G. 1995a, A\&A, 296,217

Blackwell, D. E., Smith, G., \& Lynas-Gray, A. E. 1995b, A\&A, 303,575

Böhm-Vitense, E. 1958, Z. Astrophys., 46, 108

Boyarchuk, A. A., Lyubimkov, L. S., \& Sakhibullin, N. A. 1985, Astrofizika, 22, 339

Butler, K., \& Giddings, J. 1985, Newsletter on Analysis of Astronomical spectra No. 9, University of London

Canuto, V. M., \& Mazzitelli, I. 1992, ApJ, 389, 72

Castelli, F., Gratton, R. G., \& Kurucz, R. L. 1997, A\&A, 318, 841

Drawin, H. W. 1968, Z. Physik, 211, 404

Drawin, H. W. 1969, Z. Physik, 225, 483

Freytag, B., Ludwig, H.-G., \& Steffen, M. 1996, A\&A, 313, 497

Fuhr, J. R., Martin, G. A., \& Wiese, W. L. 1988, J. Phys. Chem. Ref. Data, 17, Suppl. 4

Fuhrmann, K. 1998, A\&A, 338, 161

Fuhrmann, K. 2000, A\&A, in press

Fuhrmann, K., Pfeiffer, M., Frank, C., Reetz, J., \& Gehren, T. 1997, A\&A, 323, 909

Gehren, T., Reile, C., \& Steenbock, W. 1991, in Stellar Atmospheres: Beyond Classical Models, ed. L. Crivellari, I. Hubeny, \& D. G. Hummer (Kluwer, Dordrecht) 387

Gratton, R. G., Carretta, E., Eriksson, K., \& Gustafsson, B. 1999, A\&A, 350, 955

Gray, D. F. 1977, ApJ, 218, 530

Hannaford, P., Lowe, R. M., Grevesse, N., \& Noels, A. 1992, A\&A, 259, 301

Holweger, H. 1967, Zs.f.Ap., 65, 365

Holweger, H., \& Müller, E. A. 1974, Sol. Phys., 39, 19

Holweger, H., Heise, C., \& Kock, M. 1990, A\&A, 232, 510

Holweger, H., Bard, A., Kock, M., \& Kock, A. 1990, A\&A, 232, 510

Holweger, H., Kock, M., \& Bard, A. 1995, A\&A, 296, 233
Hummer, D. G., Berrington, K. A., Eissner, W., et al. 1993, A\&A, 279, 298

Johansson, S., Nave, G., Geller, M., et al. 1994, ApJ, 429, 419

Kaulakys, B. 1985, J. Phys. B, 18, L167

Kostik, R. I., Shchukina, N. G., \& Rutten, R. J. 1996, A\&A, 305,325

Kupka, F. 1999, ApJ, 526, L45

Kurucz, R. L. 1992, Rev. Mex. Astron. Astrof., 23, 45

Kurucz, R. L., Furenlid, I., Brault, J., \& Testerman, L. 1984, Solar Flux Atlas from 296 to 1300 nm, Kitt Peak National Solar Observatory

Leininger, T., Gadéa, F. X., \& Dickinson, A. S. 2000, J. Phys. B: At. Mol. Opt. Phys., 33, 1805

Lites, B. W. 1972, Observation and Analysis of the Solar Neutral Iron Spectrum, Ph.D. Thesis, Boulder

Lites, B. W. 1973, Sol. Phys., 32, 283

Lites, B. W., \& Cowley, C. R. 1974, A\&A, 31, 361

Lyubimkov, L. S., \& Boyarchuk, A. A. 1983, Astrofizika, 19, 683

Magain, P. 1988, in The Impact of High S/N Spectroscopy on Stellar Physics, ed. G. Cayrel de Strobel, \& M. Spite (Reidel, Dordrecht) 485

Magain, P., \& Zhao, G. 1996, A\&A, 305, 245

Mashonkina, L., Gehren, T., \& Bikmaev, I. 1999, A\&A, 343, 519

Mashonkina, L., \& Gehren, T. 2000, A\&A, submitted

Meylan, T., Furenlid, I.,Wiggs, M. S., \& Kurucz, R. L. 1993, ApJS, 85, 163

Mihalas, D., Heasley, J. N., \& Auer, L. H. 1975, NCR Techn. Note, STR-104, Boulder

Nave, G., Johansson, S., Learner, R. C. M., Thorne, A. P., \& Brault, J. W. 1994, ApJS, 94, 221

Nissen, P. E., Høg, E., \& Schuster, W. J. 1997, in Hipparcos Venice '97 Symp. (ESA SP-402), ESA, Nordwijk

Nordlund, Å., Spruit, H. C., Ludwig, H.-G., \& Trampedach, R. 1997, A\&A, 328, 229

Pelan, J., \& Berrington, K. A. 1997, A\&AS, 122, 177

Raassen, A. J. J., \& Uylings, P. H. M. 1998, A\&A, 340, 300

Schnabel, R., Kock, M., \& Holweger, H. 1999, A\&A, 342, 610

Schoenfeld, W. G., Chang, E. S., Geller, M., et al. 1995, A\&A, 301,593

Seaton, M. J. 1962, in Atomic and Molecular Processes (Acad. Press, New York)

Sikström, C. M., et al. 1999, J. Phys. B.: At. Mol. Opt. Phys., 32,5687

Spruit, H. C., Nordlund, Å., \& Title, A. M. 1990, ARA\&A, 28, 263

Steenbock, W. 1985, in Cool Stars with Excesses of Heavy Elements, ed. M. Jaschek, \& P. C. Keenan (Reidel, Dordrecht) 231

Steenbock, W., \& Holweger, H. 1984, A\&A, 130, 319

Steffen, M., in Stellar Atmospheres: Beyond Classical Models, loc. cit., 387

Stein, R. F., \& Nordlund, Å. 1998, ApJ, 499, 914

Sugar, J., \& Corliss, C. 1985, J. Phys. Chem. Ref. Data 14, Suppl. 2

Takeda, Y. 1991, A\&A, 242, 455

Thévenin, F., \& Idiart, T. P. 1999, ApJ, 521, 753

Unsöld, A. 1968, Physik der Sternatmosphären (SpringerVerlag, Berlin-Heidelberg-New York)

van Regemorter, H. 1962, ApJ, 136, 906

Zhao, G., Butler, K., \& Gehren, T. 1998, A\&A, 333, 219 